TRANSACTIONS OF THE

AMERICAN MATHEMATICAL SOCIETY

Volume 352, Number 9, Pages 4171-4194

S 0002-9947(00)02163-2

Article electronically published on May 22, 2000

\title{
THE CONLEY INDEX OVER A BASE
}

\author{
MARIAN MROZEK, JAMES F. REINECK, AND ROMAN SRZEDNICKI
}

\begin{abstract}
We construct a generalization of the Conley index for flows. The new index preserves information which in the classical case is lost in the process of collapsing the exit set to a point. The new index has most of the properties of the classical index. As examples, we study a flow with a knotted orbit in $\mathrm{R}^{3}$, and the problem of continuing two periodic orbits which are not homotopic as loops.
\end{abstract}

\section{INTRODUCTION}

The Conley index is a useful tool in the study of flows. To obtain the index of an isolated invariant set $S$, one takes an index pair $\left(P_{1}, P_{2}\right)$ for $S$ and collapses $P_{2}$ to a point. (Recall that $P_{1}$ is an isolating neighborhood for $S$, and $P_{2}$ is a subset of $P_{1}$ such that each trajectory which leaves $P_{1}$ passes through $P_{2}$.) The homotopy type of the resulting quotient space is independent of the choice of index pair. Indeed, given two index pairs, after collapsing the exit sets there is a flow-defined homotopy equivalence between the resulting quotient spaces. The homotopy type of the quotient space, or more precisely, the collection of all such quotients and homotopy equivalences, is called the Conley index of $S$, and is denoted $h(S)$.

One weakness of the index is that information may be lost when $P_{2}$ is collapsed to a point. An example from Conley's monograph [Co, II.2.4] illustrates this.

In Figure 1. $P_{1}$ is a cylinder from which a tubular neighborhood of a knotted orbit has been removed, and $P_{2}$ is the punctured disc at the bottom of $P_{1}$. $P_{2}$ is not a strong deformation retract of $P_{1}$, so by Ważewski's theorem, there is a point whose forward orbit is contained in $P_{1}$, and the omega-limit set of this orbit is an invariant set contained in $P_{1}$. However, when $P_{2}$ is collapsed to a point, the resulting quotient space is homotopic to a point, and thus the Conley index does not detect the invariant set. Indeed, a question posed in Co, IV.8.B, C] asks whether there exists a finer invariant, independent of the choice of index pairs, which retains some information lost under the collapse.

The Conley index is a continuation invariant. If one has an isolating neighborhood $N$, and deforms the flow in such a way that $N$ remains an isolating neighborhood throughout the deformation, then the sets isolated by $N$ are related by continuation, and have the same index. An obvious question is the converse: if two sets have the same index, are they related by continuation? It is easy to find

Received by the editors May 3, 1996 and, in revised form, March 10, 1997.

2000 Mathematics Subject Classification. Primary 37B30; Secondary 55R70, 37B35, 54H20.

Key words and phrases. Isolated invariant set, Conley index, continuation, fiberwise pointed space.

The first author was supported by KBN, Grant 0449/P3/94/06.

The third author was supported by KBN, Grant 2 P03A 04010. 


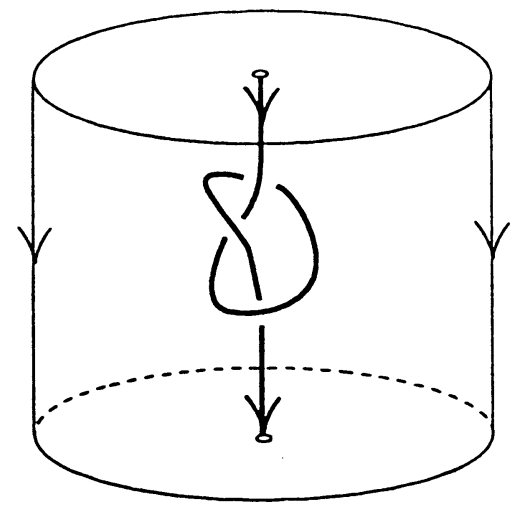

FiguRE 1. A knotted orbit in $\mathrm{R}^{3}$

examples to show that this need not be the case. For example, if the phase space is disconnected, it is easy to construct flows with critical points of the same index in different components, and these sets are not related by continuation. The problem here is that the index does not take into account how the set lies in the phase space. In the example with a disconnected phase space, it is obvious that there is no continuation, but a more subtle question is the following: can two periodic orbits which are not homotopic as loops be related by continuation? A repelling periodic orbit in the punctured plane has the same index as the disjoint union of two critical points, so it is not so clear that there is no continuation between periodic orbits in different homotopy classes.

In this paper, we generalize the index by gluing the exit set $P_{2}$ of a regular index pair to a topological space $Z$, which we call the base space. The gluing map $\omega: X \rightarrow Z$ is defined on the phase space $X$, and the index of a set $S$ over $Z$ is the so-called fiberwise deforming homotopy type of the adjunction space $P_{1} \cup_{\left.\omega\right|_{P_{2}}} Z$. The precise definition of fiberwise deforming homotopy type is given below, but it is a finer invariant than the usual homotopy type of the adjunction, as Example 8.2 illustrates. If $Z$ is a point, then this gives the classical Conley index. Another possibility for an arbitrary flow is $Z=X, \omega=\mathrm{id}_{X}$. These two examples represent the maximal and minimal information loss due to collapsing. Other spaces $Z$ and maps $\omega$ lie somewhere in between in terms of information lost upon collapse.

The index over a base has properties similar to the classical Conley index. The fiberwise deforming homotopy type of the quotient $P_{1} \cup_{\left.\omega\right|_{P_{2}}} Z$ is independent of the choice of regular index pair, and is invariant under continuation. The construction of the index over a base is fully functorial. In particular, this means that the new index extends to a connected simple system, and one may also obtain a Conley functor as in $\mathrm{KM}$. By making a judicious choice of $Z$ and $\omega$, one can often obtain more information than in the classical case. For example, in the flow from Figure 1 if one chooses $Z$ to be $P_{1}$, and $\omega$ to be the identity, then the space $P_{1} \cup_{\left.\omega\right|_{P_{2}}} Z$ is not homotopically equivalent to $Z$, hence the index over $Z$ of the maximal invariant set $S$ inside of $P_{1}$ is nontrivial. This fact not only means that $S \neq \varnothing$ (like the theorem of Ważewski), but implies that the nearby continuations of $S$ are also nonempty.

In the case of periodic orbits in different homotopy classes, let $X$ denote the punctured plane $\mathrm{R}^{2} \backslash\{0\}, Z$ be the unit circle, and $\omega(x)=x /|x|$. Suppose $S$ is a 
repelling periodic orbit, with $P_{1}$ an annulus, and $P_{2}=\partial P_{1}$. If $S$ is homotopically nontrivial, then the index of $S$ over $Z$ is a 2-torus, but if $S$ is homotopically trivial, it is the wedge of a 2 -sphere and two 1-spheres. Thus these sets are not related by continuation in any family of flows on the punctured plane.

We were originally led to this generalization of the index by studying the classical Conley index for periodic orbits. In the case of hyperbolic periodic orbits, our generalized index has a structure similar to that of a bundle over the circle as in the noncontractible orbit in the punctured plane. Bundles over the circle are classified by a single gluing map on the fiber. In the periodic orbit case that map is related to the Poincaré map. In particular, all information carried by the discrete-time Conley index of the Poincaré map may be regained from an appropriate Conley index over $S^{1}$. This result will be described precisely in a future paper.

In this paper, our setting is the case of semiflows on locally compact metric spaces, but the same ideas can be applied to infinite dimensional semiflows such as those generated by partial differential equations in the setting studied by Rybakowski (see $[\mathrm{Ry}]$ ). Since the additional technical details needed in the infinite dimensional case would obscure the main ideas of this paper, we only deal with the finite dimensional case. However, we work with semiflows, and all the topological background is developed to work in the general case; in particular, in Section 4 we deal with pairs of closed subspaces instead of compact ones. Thus the reader familiar with the Rybakowski approach will be able to extend the theory presented here to the Rybakowski setting.

A very special case of our index has already been considered in the paper [Ba] by Bartsch; the phase space $X$ in that paper is the total space of a locally trivial bundle $p: X \rightarrow \Lambda$, and the flow is fiber-preserving. The Bartsch index of an isolated invariant set in $X$ is equal to the index defined by us with $Z=\Lambda$ and $\omega=p$.

We denote the sets of all reals, integers, nonnegative integers, and nonpositive integers by $\mathrm{R}, \mathrm{Z}, \mathrm{Z}^{+}$, and $\mathrm{Z}^{-}$, respectively. For a given topological space $X$ and $A \subset X$, we denote the closure of $A$ by cl $A$, its interior by int $A$ and its boundary by bd $A$. By $I$ we denote the closed interval $[0,1]$.

The organization of the paper is as follows. Section 2 contains main results. In Section 3 we introduce the fiberwise deforming map and homotopies between fiberwise pointed spaces and we study in detail their properties. Section 4 is devoted to fiberwise pointed spaces generated by closed pairs. Regular index pairs needed in our construction are discussed in Section 5 . The formal definition of the Conley index over a base together with the proof of its correctness is presented in Section 6] In Section 7 we prove the main properties of the Conley index over a base. The last section contains examples.

The concept of the fiberwise deforming homotopy required in our construction seems to be a new idea. Therefore we devoted a substantial part of the paper (Sections 3 and 4) to it. In order to understand only the construction of the Conley index over a base the reader may prefer to skip Section 3 beyond Proposition 3.3 and Section 4 beyond Proposition 4.3 in the first reading.

\section{Main Results}

In this section we state our main results. We assume the reader is familiar with the standard concepts of Conley index theory as presented in $[\mathrm{Co}, \mathrm{Sm}]$. Precise definitions are given later. Let $X$ be a locally compact metric space and let $\varphi$ be a 
semiflow on $X$. For an index pair $P=\left(P_{1}, P_{2}\right)$ in a neighborhood of some isolated invariant set, define the map $\tau_{P}: P_{1} \rightarrow[0, \infty]$ by

$$
\tau_{P}(x):= \begin{cases}\sup \left\{t \geq 0 \mid x \cdot[0, t] \subset P_{1} \backslash P_{2}\right\} & \text { if } x \in P_{1} \backslash P_{2}, \\ 0 & \text { if } x \in P_{2} .\end{cases}
$$

Recall that an index pair in a flow is called regular if $\tau_{P}$ is continuous. In the case of semiflows the definition is somewhat more complicated, and is given in Section 5 . A pair $\left(B, B^{-}\right)$consisting of an isolating block $B$ and its exit set $B^{-}$is an example of regular index pair. Every neighborhood of an isolated invariant set contains a regular index pair.

Let $Z$ be a Hausdorff space and $\omega: X \rightarrow Z$ be a continuous map. For the regular index pair $P=\left(P_{1}, P_{2}\right)$ we define $U_{\omega}(P)$ as the adjunction $P_{1} \cup_{\left.\omega\right|_{P_{2}}} Z$, i.e.

$$
U_{\omega}(P):=Z \times 0 \cup P_{1} \times 1 / \sim,
$$

where $\sim$ denotes the minimal equivalence relation which identifies a point $(x, 1) \in$ $P_{2} \times 1$ with $(\omega(x), 0) \in Z \times 0$. Denote by $[u, i]$ the equivalence class of $(u, i)$ in $U_{\omega}(P)$. There are a natural embedding $s_{P}: Z \rightarrow U_{\omega}(P), z \mapsto[z, 0]$ and a natural projection $r_{P}: U_{\omega}(P) \rightarrow Z ; r_{P}([x, 1])=\omega(x)$ and $r_{P}([z, 0])=z$. Obviously, $r_{P} \circ s_{P}=\mathrm{id}_{Z}$.

As in the case of the classical Conley index theory, the homotopy type of $U_{\omega}(P)$ is independent of the choice of (regular) index pair. In fact, a stronger result is true. In order to state it, we introduce the notion of fiberwise deforming homotopy type over $Z$ (its detailed description will be presented in Section [3). Let $(U, r, s)$ be a triple consisting of a topological space $U$ and continuous maps $r: U \rightarrow Z$ and $s: Z \rightarrow U$ such that $r \circ s=\mathrm{id}_{Z}$. Following [J3] we call such a triple a fiberwise pointed space over $Z$. If $Z$ is a one-point space, then this notion coincides with the notion of a topological space with a base-point. As we have shown above, $\left(U_{\omega}(P), r_{P}, s_{P}\right)$ is a fiberwise pointed space. Two fiberwise pointed spaces $(U, r, s)$ and $\left(U^{\prime}, r^{\prime}, s^{\prime}\right)$ are equivalent if there exist continuous maps $f: U \rightarrow U^{\prime}$ and $f^{\prime}: U^{\prime} \rightarrow U$ satisfying

$$
\begin{gathered}
f \circ s=s^{\prime}, \quad f^{\prime} \circ s^{\prime}=s, \\
r^{\prime} \circ f \simeq r \operatorname{rel} s(Z), \quad r \circ f^{\prime} \simeq r^{\prime} \operatorname{rel} s^{\prime}(Z), \\
f^{\prime} \circ f \simeq \operatorname{id}_{U} \operatorname{rel} s(Z), \quad f \circ f^{\prime} \simeq \operatorname{id}_{U^{\prime}} \operatorname{rel} s^{\prime}(Z) .
\end{gathered}
$$

The equivalence class is called the fiberwise deforming homotopy type over $Z$ (in contrast to the notion of fiberwise pointed homotopy type introduced in [J] where it is assumed additionally that $r^{\prime} \circ f=r$ and $\left.r \circ f^{\prime}=r^{\prime}\right)$. The proof of the following theorem is postponed to Section [6]

Theorem 2.1. If $P$ and $Q$ are two regular index pairs for an isolated invariant set $S$, then $\left(U_{\omega}(P), r_{P}, s_{P}\right)$ and $\left(U_{\omega}(Q), r_{Q}, s_{Q}\right)$ have the same fiberwise deforming homotopy type over $Z$.

The fiberwise deforming homotopy type over $Z$ described in Theorem 2.1 is denoted by $h_{\omega}(S, \varphi)$ and is called the Conley index of $S$ over the base $Z$. In particular, the index over a one-point space, up to a natural identification, is equal to the classical Conley index $h(S, \varphi)$. Of course, the index over $Z$ also depends on the map $\omega$ (called the base map), hence the notation. A detailed discussion on the dependence on the base map and relations to the classical index is postponed to Section [7. Now we concentrate on the results which indicate that the new index 
has all of the basic properties of the classical one. Proofs of the theorems contained in the remaining part of this section can be found in Section 7

Let us call the fiberwise deforming homotopy type of $\left(Z, \mathrm{id}_{Z}, \mathrm{id}_{Z}\right)$ trivial and let us denote it by $\overline{0}_{Z}$. A direct consequence of the introduced notions is

Theorem 2.2. $h_{\omega}(\varnothing, \varphi)=\overline{0}_{Z}$.

The wedge sum $\vee$ of fiberwise deforming homotopy types is defined in an obvious way (compare Section 3), hence we can state the additivity property of the index:

Theorem 2.3. Suppose $S$ and $S^{\prime}$ are disjoint isolated invariant sets. Then

$$
h_{\omega}\left(S \cup S^{\prime}, \varphi\right)=h_{\omega}(S, \varphi) \vee h_{\omega}\left(S^{\prime}, \varphi\right) .
$$

On the other hand, the smash product $\vee$ of fiberwise deforming homotopy types is not defined in general; in order to define it correctly one should consider fiberwise well-pointed spaces over $Z$, i.e. fiberwise pointed spaces $(U, r, s)$ such that $s$ is a cofibration (see Section 3). Actually, if $P$ is a regular index pair, then $\left(U_{\omega}(P), r_{P}, s_{P}\right)$ is well-pointed (Proposition 6.1), hence we are able to establish the multiplicativity property of the index: Let $\varphi$ and $\omega$ be as above and let $\varphi^{\prime}$ be a semiflow on $X^{\prime}$. By $\varphi^{\#}$ denote the product flow on $X \times X^{\prime}$, i.e. $\varphi_{t}^{\#}\left(x, x^{\prime}\right)=\left(\varphi_{t}(x), \varphi_{t}^{\prime}\left(x^{\prime}\right)\right)$. Let $Z^{\prime}$ be another Hausdorff space and let $\omega^{\prime}: X^{\prime} \rightarrow Z^{\prime}$ be a continuous map.

Theorem 2.4. Assume that $S$ and $S^{\prime}$ are isolated invariant sets; $S$ for $\varphi$ and $S^{\prime}$ for $\varphi^{\prime}$. Then

$$
h_{\omega \times \omega^{\prime}}\left(S \times S^{\prime}, \varphi^{\#}\right)=h_{\omega}(S, \varphi) \wedge h_{\omega^{\prime}}\left(S^{\prime}, \varphi^{\prime}\right) .
$$

As in the classical case, the index over $Z$ is invariant under continuation. Consider a family of semiflows $\varphi^{\lambda}: X \times \mathrm{R}^{+} \rightarrow X$ continuously depending on $\lambda \in I$. We have the following theorem.

Theorem 2.5. If $N$ is an isolating neighborhood with respect to $\varphi^{\lambda}$ for all $\lambda \in I$ and $S^{\lambda}$ is the maximal invariant set of $\varphi^{\lambda}$ inside $N$, then $h_{\omega}\left(S^{\lambda}, \varphi^{\lambda}\right)$ does not depend on $\lambda$.

We can now present the example with a knotted periodic orbit from the Introduction in more detail.

Example 2.6. Consider the flow $\varphi$ pictured in Figure 1 Let the phase space $X$ of the flow be $\mathrm{R}^{3}$ minus the knotted curve. Let the index pair be $P=\left(P_{1}, P_{2}\right)$, let $P_{1}$ be the cylinder (with the bases at the levels $z=0$ and $z=1$ ) minus a tubular neighborhood of the curve, and let $P_{2}$ be the punctured disc at the base in the level $z=0$ of $P_{1}$. Put $S=\operatorname{Inv} P_{1}$. Let $Z=P_{1}$ and $\omega$ be the natural strong deformation retraction of $X$ onto $P_{1}$, i.e. $\left.\omega\right|_{P_{1}}$ is the identity and $\omega_{X \backslash P_{1}}$ projects the point $(x, y, z)$ onto $(x, y, 0)$ if $z \leq 0$, onto $(x, y, 1)$ if $z \geq 1$, and then retracts (via the natural retraction in the tubular neighborhood) all the points with $z \in[0,1]$ onto the boundary of $P_{1}$. $\mathbf{U}_{\omega}(P)$ consists of two copies of $P_{1}$ glued in $P_{2}$. Its fundamental group is equal to the fundamental group of the doubled knot, hence it is different from the fundamental group of $Z$ (i.e. the group of the knot, see [Ro]), hence the index $h_{\omega}(S, \varphi)$ of $S=\operatorname{Inv} P_{1}$ is not trivial. In particular, it follows that any continuation of $S$ is also nonempty — this fact cannot be directly obtained from the Ważewski Theorem.

More examples will be presented in the last section. 


\section{FiberWise POINTED SPACES OVER A BASE}

In the sequel, $Z$ denotes a fixed topological space. A triple $\mathbf{U}=(U, r, s)$ is called a fiberwise pointed (topological) space over $Z$ if $U$ is a topological space, $r: U \rightarrow Z$ and $s: Z \rightarrow U$ are continuous maps, and $r \circ s=\mathrm{id}_{Z}$ (compare [J3, p. 41-42]). In [J1 p. 163] and [J2, p. 98] such a space is also called a sectioned space over $Z$. We refer to $Z$ as the base space, to $r$ as the projection, and to $s$ as the section of $\mathbf{U}$. The projection is an $r$-map in the sense of [Bo. In particular it follows that $s(Z)$ is a retract (hence a closed subset) of $U, s \circ r: U \rightarrow s(Z)$ is a retraction, and $s$ and the restriction of $r$ are mutually inverse homeomorphisms between $Z$ and $s(Z)$.

We want to turn the fiberwise pointed spaces into a category. Let $\mathbf{U}^{\prime}=\left(U^{\prime}, r^{\prime}, s^{\prime}\right)$ be another fiberwise pointed space over $Z$. A natural choice for a morphism from $\mathbf{U}$ to $\mathbf{U}^{\prime}$ is to take a fiberwise pointed map, i.e. a continuous map $\phi: U \rightarrow U^{\prime}$ such that $r^{\prime} \circ \phi=r$ and $\phi \circ s=s^{\prime}$ (comp. [J1, [J3] for instance). For our purposes such a definition is too restrictive.

We define a fiberwise deforming map (shortly: an f.d. map) $f: \mathbf{U} \rightarrow \mathbf{U}^{\prime}$ as a continuous map $f:(U, s(Z)) \rightarrow\left(U^{\prime}, s^{\prime}(Z)\right)$ such that

$$
r^{\prime} \circ f \simeq r \operatorname{rel} s(Z) \text {. }
$$

Obviously, a fiberwise pointed map is an f.d. map. Note that there always exists a fiberwise pointed map (and consequently an f.d. map) between $\mathbf{U}$ and $\mathbf{U}^{\prime}$, namely $s^{\prime} \circ r$ (it is called the fiberwise constant map).

Proposition 3.1. If $f: \mathbf{U} \rightarrow \mathbf{U}^{\prime}$ is f.d., then $f \circ s=s^{\prime}$ and the restriction of $f$

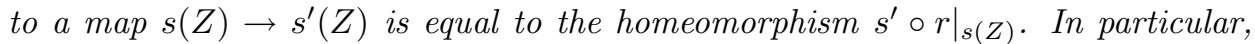
$f(s(Z))=s^{\prime}(Z)$.

One can easily verify that the identity map is an f.d. map and the composition of f.d. maps is an f.d. map. Thus fiberwise pointed spaces over $Z$ together with f.d. maps constitute a category. We will call it the fiberwise deforming category and we will denote it by $\mathrm{Fib}_{Z}$.

We define $\mathbf{U} \vee \mathbf{U}^{\prime}$, the fiberwise pointed wedge, as the triple $\left(U \vee U^{\prime}, r_{\vee}, s_{\vee}\right)$ such that $U \vee U^{\prime}$ is the disjoint union of $U$ and $U^{\prime}$ with $s(z)$ and $s^{\prime}(z)$ identified, i.e. $U \vee U^{\prime}:=U \times 0 \cup U^{\prime} \times 1 / \sim_{\vee}$, where $\sim_{\vee}$ denotes the equivalence relation generated by $(s(z), 0) \sim_{\vee}\left(s^{\prime}(z), 1\right)$ for any $z \in Z$. The maps $r_{\vee}, s_{\vee}$ are given by

$$
r_{\vee}([x, i]):= \begin{cases}r(x) & \text { if } x \in U, \\ r^{\prime}(x) & \text { if } x \in U^{\prime},\end{cases}
$$

and $s_{\vee}(z):=[(s(z), 0)]=\left[\left(x^{\prime}(z), 0\right)\right]$. It is easy to check that $r_{\vee}$ is well-defined, that $\mathbf{U} \vee \mathbf{U}^{\prime}$ is a fiberwise pointed space, and that this agrees with the usual definition of wedge when $Z$ is a single point.

Let $\mathbf{U}=(U, r, s)$ and $\mathbf{U}^{\prime}=\left(U^{\prime}, r^{\prime}, s^{\prime}\right)$ be two fiberwise pointed spaces over $Z$ and $Z^{\prime}$, respectively. In the cartesian product $U \times U^{\prime}$ define an equivalence relation $\sim_{\wedge}$ by $\left(u, u^{\prime}\right) \sim_{\wedge}\left(v, v^{\prime}\right)$ if $\left(u, u^{\prime}\right)=\left(v, v^{\prime}\right)$ or $u=v \in s(Z), r^{\prime}\left(u^{\prime}\right)=r^{\prime}\left(v^{\prime}\right)$ or $r(u)=r(v), u^{\prime}=v^{\prime} \in s^{\prime}(Z)$. Put

$$
U \wedge U^{\prime}:=U \times U^{\prime} / \sim \wedge
$$

and denote by $u \wedge u^{\prime}$ the equivalence class of $\left(u, u^{\prime}\right)$. Define maps $r_{\wedge}: U \wedge U^{\prime} \rightarrow Z \times Z^{\prime}$ and $s_{\wedge}: Z \times Z^{\prime} \rightarrow U \wedge U^{\prime}$ by

$$
r_{\wedge}\left(u \wedge u^{\prime}\right):=\left(r(u), r^{\prime}\left(u^{\prime}\right)\right), \quad s_{\wedge}\left(z, z^{\prime}\right):=s(z) \wedge s^{\prime}\left(z^{\prime}\right) .
$$


The triple

$$
\mathbf{U} \wedge \mathbf{U}^{\prime}:=\left(U \wedge U^{\prime}, r_{\wedge}, s_{\wedge}\right)
$$

is a fiberwise pointed space over $Z \times Z^{\prime}$ and is called the fiberwise pointed smash product of $\mathbf{U}$ and $\mathbf{U}^{\prime}$. Again, if both $Z$ and $Z^{\prime}$ are one-pointed spaces, then this notion agrees with the usual smash product.

By the homotopy in the category $\mathrm{Fib}_{Z}$ we mean a collection of f.d. maps $F_{t}$ : $\mathbf{U} \rightarrow \mathbf{U}^{\prime}, t \in I$ such that the map $F: U \times I \ni(x, t) \rightarrow F_{t}(x) \in U^{\prime}$ is continuous. We say that two f.d. maps $f, g: \mathbf{U} \rightarrow \mathbf{U}^{\prime}$ are homotopic in $\mathrm{Fib}_{Z}$ if there exists a homotopy $F_{t}: \mathbf{U} \rightarrow \mathbf{U}^{\prime}, t \in I$ in $\mathrm{Fib}_{Z}$ such that $F_{0}=f, F_{1}=g$.

As an easy consequence of Proposition 3.1 we obtain the following:

Proposition 3.2. Assume $f: \mathbf{U} \rightarrow \mathbf{U}^{\prime}$ is an f.d. map and $g: U \rightarrow U^{\prime}$ is continuous. If

$$
f \simeq g \operatorname{rel} s(Z)
$$

then $g$ is also an f.d. map from $\mathbf{U}$ to $\mathbf{U}^{\prime}$ and $f$ and $g$ are homotopic in $\mathrm{Fib}_{Z}$.

Proof. We have $f \simeq g \operatorname{rel} s(Z)$, thus also $r^{\prime} \circ f \simeq r^{\prime} \circ g \operatorname{rel} s(Z)$. Since $f$ is an f.d. map, $r \simeq r^{\prime} \circ f \simeq r^{\prime} \circ g \operatorname{rel} s(Z)$. This shows that also $g$ is an f.d. map. Let $F_{t}$ be the homotopy joining $f$ to $g$. Then $F_{t} \simeq f \operatorname{rel} s(Z)$ for each $t \in I$. It follows from what we have already proved that $F_{t}$ is an f.d. map and consequently $f$ and $g$ are homotopic in $\mathrm{Fib}_{Z}$.

Obviously, the homotopy in the category $\mathrm{Fib}_{Z}$ induces an equivalence relation between the f.d. maps. Taking equivalence classes of f.d. maps as a new collection of morphisms we obtain another category, which we will call the fiberwise deforming homotopy category. We will denote it by Htp Fib . Obviously, we have also the homotopy functor Htp $: \mathrm{Fib}_{Z} \rightarrow \mathrm{HtpFib}_{Z}$ sending each f.d. map to its equivalence class. An f.d. map $\phi$ will be called a fiberwise deforming homotopy equivalence if $\operatorname{Htp}(\phi)$ is an isomorphism in $\mathrm{HtpFib}_{Z}$.

Proposition 3.3. Assume $f: \mathbf{U} \rightarrow \mathbf{U}^{\prime}$ is an f.d. map. If there exists a continuous map $f^{\prime}: U^{\prime} \rightarrow U$ such that

$$
f^{\prime} \circ f \simeq \operatorname{id}_{U} \operatorname{rel} s(Z), f \circ f^{\prime} \simeq \operatorname{id}_{U^{\prime}} \operatorname{rel} s^{\prime}(Z),
$$

then $f^{\prime}$ is also an f.d. map and $f$ and $f^{\prime}$ are mutually inverse fiberwise deforming homotopy equivalences.

Proof. First observe that $f^{\prime} \circ f \simeq \operatorname{id}_{U} \operatorname{rel} s(Z)$ implies that $f^{\prime} f(s(Z))=s(Z)$. From the homotopy equivalence $f \circ f^{\prime} \simeq \operatorname{id}_{U} \operatorname{rel} s(Z)$ we get $r^{\prime} \circ f \circ f^{\prime} \simeq r^{\prime} \operatorname{rel} s\left(Z^{\prime}\right)$ and $r \circ f^{\prime} \simeq r^{\prime}$ rel $s^{\prime}(Z)$, because $f$ is an f.d. map. It follows that $f^{\prime}$ is also an f.d. map and consequently $f$ and $f^{\prime}$ are mutually inverse fiberwise deforming homotopy equivalences.

We say that two fiberwise pointed spaces $\mathbf{U}$ and $\mathbf{U}^{\prime}$ over $Z$ have the same fiberwise deforming homotopy type over $Z$ if there exists a fiberwise deforming homotopy equivalence between them. The equivalence class of spaces with the same fiberwise deforming homotopy type as $\mathbf{U}$ is denoted by $[\mathbf{U}]_{Z}$. A distinguished role is played by

$$
\overline{0}_{Z}:=\left[\left(Z, \mathrm{id}_{Z}, \mathrm{id}_{Z}\right)\right]_{Z}
$$

the trivial fiberwise deforming homotopy type over $Z$. 
We compare various homotopy types on a simple example.

Example 3.4. For $x \in \mathrm{R} \backslash 0$ put $V_{x}=(\mathrm{R} \backslash 0) \times 0 \cup\{x\} \times 1$, i.e. $V_{x}$ is the disjoint union of $\mathrm{R} \backslash 0$ and $\{x\}$. Define $r_{x}: V_{x} \rightarrow \mathrm{R} \backslash 0$ and $s_{x}: \mathrm{R} \backslash 0 \rightarrow V_{x}$ by $r_{x}(y, i)=y$ and $s_{x}(z)=(z, 0)$. Then $\mathbf{V}_{x}=\left(V_{x}, r_{x}, s_{x}\right)$ is a fiberwise pointed space over $\mathrm{R} \backslash 0$. Each of the spaces $V_{x}$ has the same (ordinary) homotopy type of a three-point space. It is easy to see, that for $x \neq x^{\prime}$ there is no fiberwise pointed map $\mathbf{V}_{x} \rightarrow \mathbf{V}_{x^{\prime}}$ except for the constant one, hence the fiberwise pointed homotopy types (i.e. the homotopy types in the sense of [J1, J3] ) of $\mathbf{V}_{x}$ are all different. On the other hand, there are no nonconstant f.d. maps between $\mathbf{V}_{x}$ and $\mathbf{V}_{x^{\prime}}$ only in the case where the signs of $x$ and $x^{\prime}$ are opposite, hence there are exactly two different fiberwise deforming homotopy types, and $\left[\mathbf{V}_{x}\right]_{\mathrm{R} \backslash 0}=\left[\mathbf{V}_{x^{\prime}}\right]_{\mathrm{R} \backslash 0}$ iff $x x^{\prime}>0$.

In the above example we compared the fiberwise pointed homotopy types of spaces with several connected components. This can be generalized to the following simple observation.

Proposition 3.5. Let $\left\{T_{i}\right\}_{i=0, \ldots, r}$ and $\left\{T_{i}^{\prime}\right\}_{i=0, \ldots, r}$ be the sets of path components of $U$ and $U^{\prime}$, respectively. Let

$$
s(Z) \subset T_{0}, s^{\prime}(Z) \subset T_{0}^{\prime} .
$$

If $f: \mathbf{U} \rightarrow \mathbf{U}^{\prime}$ is a fiberwise deforming homotopy equivalence, then there is a permutation $\sigma, \sigma(0)=0$, such that for $i=0, \ldots, r: f\left(T_{i}\right) \subset T_{\sigma(i)}^{\prime}$ and the restriction $f_{i}: T_{i} \rightarrow T_{\sigma(i)}$ of $f$ is a homotopy equivalence such that $\left.r^{\prime} \circ f_{i} \simeq r\right|_{T_{i}}$.

In order to distinguish fiberwise deforming homotopy types of $\mathbf{U}$ and $\mathbf{U}^{\prime}$ one should compare the homotopy types $[U]$ and $\left[U^{\prime}\right]$ first. If they are equal, further necessary information can be provided by Proposition 3.5 as the next example shows:

Example 3.6. Let $W_{1}=W_{2}=S^{1} \times 0 \cup S^{1} \times 1$, i.e. $W_{1}$ and $W_{2}$ are equal to the disjoint union of two copies of the circle $S^{1}=\{x \in C:|x|=1\}$. Define $r_{i}: W_{i} \rightarrow S^{1}$ and $s_{i}: S^{1} \rightarrow W_{i}, i=1,2$ by

$$
\begin{gathered}
r_{1}(x, i)=x, \quad s_{1}(y)=(y, 0), \\
r_{2}(x, 0)=x, \quad r_{2}(x, 1)=x^{2}, \quad s_{2}(y)=(y, 0) .
\end{gathered}
$$

Then $\mathbf{W}_{i}=\left(W_{i}, r_{i}, s_{i}\right), i=1,2$ are fiberwise pointed spaces over $S^{1}$. It follows by Proposition 3.5 that if $f: \mathbf{W}_{1} \rightarrow \mathbf{W}_{2}$ is a fiberwise pointed homotopy equivalence, then $f\left(S^{1} \times 1\right) \subset\left(S^{1} \times 1\right)$ and $\left.\left.r_{2} \circ f\right|_{S^{1} \times 1} \simeq r_{1}\right|_{S^{1} \times 1}$, which is impossible. Thus $\left[\mathbf{W}_{1}\right]_{S^{1}} \neq\left[\mathbf{W}_{2}\right]_{S^{1}}$.

Remark 3.7. If we consider the question of comparison of $[\mathbf{U}]_{Z}$ and $\left[\mathbf{U}^{\prime}\right]_{Z}$ on the homotopy level, one would expect that the information provided by the triples $(H(U), H(r), H(s))$ and $\left(H\left(U^{\prime}\right), H\left(r^{\prime}\right), H\left(s^{\prime}\right)\right)$ is essentially better than the one provided by $H(U)$ and $H\left(U^{\prime}\right)$ alone ( $H$ denotes a homology functor). However, this is not the case, at least in reasonable situations: if $H(U) \cong H\left(U^{\prime}\right)$ and $H(Z)$ 
is a finitely generated abelian group, then the diagram

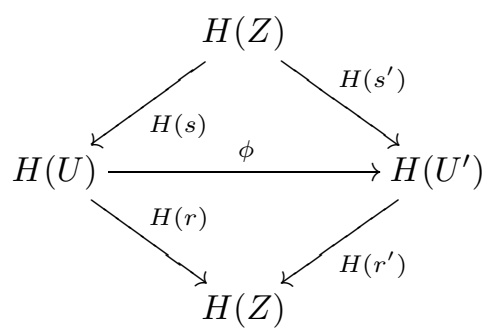

is commutative for some isomorphism $\phi$.

Indeed, let $j: U \hookrightarrow(U, s(Z))$ and $j^{\prime}: U^{\prime} \hookrightarrow\left(U^{\prime}, s^{\prime}(Z)\right)$ be the inclusions. Since $s(Z)$ is a retract of $U$, the exact sequence

$$
0 \rightarrow H(Z) \stackrel{H(s)}{\longrightarrow} H(U) \stackrel{H(j)}{\longrightarrow} H(U, s(Z)) \rightarrow 0
$$

splits and $(H(r), H(j)): H(U) \rightarrow H(Z) \oplus H(U, s(Z))$ is an isomorphism. Up to this isomorphism $H(r)$ and $H(s)$ are represented as the maps

$$
\begin{aligned}
& H(Z) \oplus H(U, s(Z)) \ni(u, v) \rightarrow u \in H(Z), \\
& H(Z) \ni u \rightarrow(u, 0) \in H(Z) \oplus H(U, s(Z)),
\end{aligned}
$$

respectively. The same holds true for the triple $\left(H\left(U^{\prime}\right), H\left(r^{\prime}\right), H\left(s^{\prime}\right)\right)$. It follows by the assumption on $H(Z)$ and by [Coh] or [W] that there exists an isomorphism $\psi: H(U, s(Z)) \rightarrow H\left(U^{\prime}, s^{\prime}(Z)\right)$, and we can put

$$
\phi=\left(H\left(r^{\prime}\right), H\left(j^{\prime}\right)\right)^{-1} \circ\left(\operatorname{id}_{H(Z)} \oplus \psi\right) \circ(H(r), H(j)) .
$$

It is easily seen that if $[\mathbf{U}]_{Z}=\left[\mathbf{U}^{\prime}\right]_{Z}$ and $[\mathbf{V}]_{Z}=\left[\mathbf{V}^{\prime}\right]_{Z}$, then $[\mathbf{U} \vee \mathbf{V}]_{Z}=$ $\left[\mathbf{U}^{\prime} \vee \mathbf{V}^{\prime}\right]_{Z}$, hence we can define

$$
[\mathbf{U}]_{Z} \vee[\mathbf{V}]_{Z}:=[\mathbf{U} \vee \mathbf{V}]_{Z},
$$

the wedge of fiberwise deforming homotopy types. Obviously, for every space $\mathbf{U}$,

$$
[\mathbf{U}]_{Z} \vee \overline{0}_{Z}=\overline{0}_{Z} \vee[\mathbf{U}]_{Z}=[\mathbf{U}]_{Z}
$$

On the other hand, we cannot provide a general definition of smash product of fiberwise deforming homotopy types, as the following example shows:

Example 3.8. Let

$$
U=I \times 0 \cup\{(0,1)\} \subset \mathrm{R}^{2}, \quad U^{\prime}=I \times 0 \cup\{(1,1)\} \subset \mathrm{R}^{2} .
$$

Assume that both $r: U \rightarrow I$ and $r^{\prime}: U^{\prime} \rightarrow I$ are given by $(x, y) \mapsto x$, and both $s: I \rightarrow U$ and $s^{\prime}: I \rightarrow U^{\prime}$ are given by $x \mapsto(x, 0)$. Let

$$
V=\left\{\frac{1}{n}: n \in \mathrm{Z}^{+}\right\} \cup\{0\} \subset \mathrm{R},
$$

$\rho: V \rightarrow\{0\}$ and $\sigma:\{0\} \rightarrow V, \sigma(0)=0$. Put $\mathbf{U}=(U, r, s), \mathbf{U}^{\prime}=\left(U^{\prime}, r^{\prime}, s^{\prime}\right)$, and $\mathbf{V}=(V, \rho, \sigma)$. One can show that $[\mathbf{U}]_{I}=\left[\mathbf{U}^{\prime}\right]_{I}$, but

$$
[\mathbf{U} \wedge \mathbf{V}]_{I \times 0} \neq\left[\mathbf{U}^{\prime} \wedge \mathbf{V}\right]_{I \times 0} .
$$

In order to verify this assertion we introduce new, simpler spaces

$$
W=I \times 0 \cup\left\{\left(0, \frac{1}{n}\right): n \in \mathrm{Z}^{+}\right\}, \quad W^{\prime}=I \times 0 \cup\left\{\left(1, \frac{1}{n}\right): n \in \mathrm{Z}^{+}\right\},
$$


mappings $R: W \rightarrow I,(x, y) \mapsto x, S: I \rightarrow W, x \mapsto(x, 0)$, and analogous mappings $R^{\prime}$ and $S^{\prime}$ for $W^{\prime}$. Put $\mathbf{W}=(W, R, S)$ and $\mathbf{W}^{\prime}=\left(W^{\prime}, R^{\prime}, S^{\prime}\right)$. The map $U \wedge V \rightarrow W$ given by $[((0,1), z)] \mapsto(0, z)$ and $[((x, 0), z)] \mapsto(x, 0)$ establishes the isomorphism (up to the identification $I=I \times 0$ ) between $\mathbf{U} \wedge \mathbf{V}$ and $\mathbf{W}$. Similarly, there is an isomorphism between $\mathbf{U}^{\prime} \wedge \mathbf{V}$ and $\mathbf{W}^{\prime}$, hence it suffices to prove that $[\mathbf{W}]_{I} \neq\left[\mathbf{W}^{\prime}\right]_{I}$.

Let $f: \mathbf{W} \rightarrow \mathbf{W}^{\prime}$ be an f.d. map. Then there is an $n_{0} \neq 0$ such that $f\left(0,1 / n_{0}\right) \in$ $I \times 0 \subset W^{\prime}$ because $f(0,0)=(0,0)$. If $f: \mathbf{W}^{\prime} \rightarrow \mathbf{W}$ is any f.d. map, then $\left(f^{\prime} \circ f\right)\left(0,1 / n_{0}\right)=f\left(0,1 / n_{0}\right)$ is contained in $I \times 0$, hence in a different path component than $\left\{\left(0,1 / n_{0}\right)\right\}$ and thus $f^{\prime} \circ f$ cannot be homotopic to the identity, hence the types must be different.

By the previous example it is clear that in order to define the smash product of fiberwise deforming homotopy types one should introduce a more restrictive class than fiberwise pointed. Recall that the pair of spaces $(X, A)$, with $A$ closed, is called cofibered if $X \times 0 \cup A \times I$ is a retract of $X \times I$ or, equivalently, if $(X, A)$ admits a Strøm structure, i.e. a pair $(G, a)$ of continuous maps $a: X \rightarrow I$ and $G: X \times I \ni(x, t) \rightarrow G_{t}(x) \in X$ such that $a^{-1}(0)=A$ and

$$
G_{0}=\operatorname{id}_{X}, \quad G_{t}(x)=x \text { if }(x, t) \in A \times I, \quad G_{1}(x) \in A \text { if } a(x)<1
$$

(compare [J2 pp. 168, 169, 172] and [Wh, pp. 22, 26]; in the latter reference the term "NDR-pair" is used).

We call $(U, r, s)$ a fiberwise well-pointed space over $Z$ provided it is fiberwise pointed and $(U, s(Z))$ is a cofibered pair (or equivalently: $s$ is a cofibration). In [J2] p. 180] such a space is called well-sectioned over $Z$.

Remark 3.9. In proofs of some results presented below, the following fact will be useful: If $\sim$ is an equivalence relation on a topological space $X$ and $H: X \times I \rightarrow Y$ is continuous such that $H(x, t)=H\left(x^{\prime}, t\right)$ if $x \sim x^{\prime}$, then the induced map $H^{\prime}$ : $(X / \sim) \times I \rightarrow Y$ given by $H ;\left([x]_{\sim}, t\right)=H(x, t)$ is continuous (compare $\left.[\mathrm{Sw}, 0.8]\right)$.

Proposition 3.10. Assume that $\mathbf{U}, \mathbf{U}^{\prime}, \mathbf{V}$, and $\mathbf{V}^{\prime}$ are fiberwise well-pointed spaces, $\mathbf{U}$ and $\mathbf{U}^{\prime}$ over $Z, \mathbf{V}$ and $\mathbf{V}^{\prime}$ over $\Omega$. If $[\mathbf{U}]_{Z}=\left[\mathbf{U}^{\prime}\right]_{Z}$ and $[\mathbf{V}]_{\Omega}=\left[\mathbf{V}^{\prime}\right]_{\Omega}$, then

$$
[\mathbf{U} \wedge \mathbf{V}]_{Z \times \Omega}=\left[\mathbf{U}^{\prime} \wedge \mathbf{V}^{\prime}\right]_{Z \times \Omega}
$$

Proof. In order to simplify notation, we assume (without loss of generality) that $\mathbf{V}=\mathbf{V}^{\prime}$. Let $\mathbf{U}=(U, r, s), \mathbf{U}^{\prime}=\left(U^{\prime}, r^{\prime}, s^{\prime}\right)$, and $\mathbf{V}=(V, \rho, \sigma)$. Let $f: \mathbf{U} \rightarrow \mathbf{U}^{\prime}$ and $f^{\prime}: \mathbf{U}^{\prime} \rightarrow \mathbf{U}$ be mutually inverse homotopy equivalences in $\mathrm{Fib}_{Z}$. We first observe that one cannot usually factor the map $(u, v) \mapsto f(u) \wedge v$ because $r(u)-r\left(u_{1}\right)$ does not imply $r^{\prime} f(u)=r^{\prime} f\left(u_{1}\right)$ in general, hence the definition of a homotopy equivalence from $\mathbf{U} \wedge \mathbf{V}$ to $\mathbf{U}^{\prime} \wedge \mathbf{V}$ must be essentially more complicated.

Denote by $h: U \times I \rightarrow Z$ a homotopy joining $r$ with $r^{\prime} \circ f$, i.e. $h_{0}=r, h_{1}=r^{\prime} \circ f$, and $h_{t} \circ s=\operatorname{id}_{Z}$ for $t \in I$. Let $h^{\prime}$ be an analogous homotopy joining $r^{\prime}$ with $r \circ f^{\prime}$. Let $(G, a),\left(G^{\prime}, a^{\prime}\right)$, and $(\Gamma, \alpha)$ be Strøm structures for $(U, s(Z)),\left(U^{\prime}, s^{\prime}(Z)\right)$, and $(V, \sigma(Z))$, respectively. For $u \in U, u^{\prime} \in U^{\prime}$, and $v \in V$ put

$$
m(u, v)=\min \{a(u), \alpha(v)\}, \quad m^{\prime}\left(u^{\prime}, v\right)=\min \left\{a^{\prime}\left(u^{\prime}\right), \alpha(v)\right\} .
$$


For $t \in(0,1]$ define maps $F_{t}: \mathbf{U} \wedge \mathbf{V} \rightarrow \mathbf{U}^{\prime} \wedge \mathbf{V}$ and $F_{t}^{\prime}: \mathbf{U}^{\prime} \wedge \mathbf{V} \rightarrow \mathbf{U} \wedge \mathbf{V}$ by

$$
\begin{gathered}
F_{t}(u \wedge v):= \begin{cases}s^{\prime} h_{\frac{4}{t} m(u, v)} G_{\frac{4}{t} m(u, v)}(u) \wedge \Gamma_{\frac{4}{t} m(u, v)}(v) & \text { if } u \in \operatorname{cl} a^{-1}\left(\left[0, \frac{1}{t} t\right)\right), \\
f G_{2-\frac{4}{t} m(u, v)}(u) \wedge \Gamma_{2-\frac{4}{t} m(u, v)}(v) & \text { or } v \in \operatorname{cl} \alpha^{-1}\left[\left[0, \frac{1}{4} t\right)\right), \\
f(u) \wedge v & \text { if } \frac{1}{4} t \leq m(u, v) \leq \frac{1}{2} t,\end{cases} \\
F_{t}^{\prime}\left(u^{\prime} \wedge v\right):= \begin{cases}s h_{\frac{4}{t} m^{\prime}\left(u^{\prime}, v\right)}^{\prime} G_{\frac{4}{t} m^{\prime}\left(u^{\prime}, v\right)}^{\prime}\left(u^{\prime}\right) \wedge \Gamma_{\frac{4}{t} m^{\prime}\left(u^{\prime}, v\right)}(v) & \text { if } u^{\prime} \in \operatorname{cl}\left(a^{\prime}\right)^{-1}\left(\left[0, \frac{1}{4} t\right)\right) \\
f^{\prime} G_{2-\frac{4}{t} m^{\prime}\left(u^{\prime}, v\right)}^{\prime}\left(u^{\prime}\right) \wedge \Gamma_{2-\frac{4}{t} m^{\prime}\left(u^{\prime}, v\right)}(v) & \text { or } v \in \operatorname{cl} \alpha^{-1}\left[\left[0, \frac{1}{4} t\right)\right), \\
f^{\prime}\left(u^{\prime}\right) \wedge v & \text { if } \frac{1}{4} t \leq m^{\prime}\left(u^{\prime}, v\right) \leq \frac{1}{2} t,\end{cases}
\end{gathered}
$$

By the definitions of smash product and Strøm structure one can verify that the above formulas give correctly defined continuous maps which are f.d. We will prove that $F_{1}$ and $F_{1}^{\prime}$ are mutually inverse homotopy equivalences. For $t \in(0,1]$ put

$$
Y_{t}=\left\{u \wedge v \in U \wedge V: m(u, v) \geq \frac{1}{2} t\right\}
$$

For $u \wedge v \in Y_{t}$ and $\tau \in I$ put

$$
n(u, v, \tau):=m^{\prime}(f(u), v)+2 \tau\left(m(u, v)-\frac{1}{2} t\right)
$$

and define maps $K_{t, \tau}: Y_{t} \rightarrow U^{\prime} \wedge V$ by

$$
\begin{aligned}
& K_{t, \tau}(u \wedge v):= \\
& \qquad \begin{array}{c}
s h_{\frac{4}{t} n(u, v, \tau)}^{\prime} G_{\frac{4}{t} n(u, v, r)}^{\prime} f(u) \wedge \Gamma_{\frac{4}{t} n(u, v, \tau)}(v) \\
\quad \text { if } f(u) \in \operatorname{cl}\left(a^{\prime}\right)^{-1}\left(\left(-\infty, \frac{1}{4} t-2 \tau\left(m(u, v)-\frac{1}{2} t\right)\right),\right. \\
f^{\prime} G_{2-\frac{1}{4} t n(u, v, \tau)}^{\prime} f(u) \wedge \Gamma_{2-\frac{4}{t} n(u, v, \tau)}(v) \\
\text { if } m^{\prime}(f(u), v) \in\left[\frac{1}{4} t-2 \tau\left(m(u, v)-\frac{1}{2} t\right), \frac{1}{2} t-2 \tau\left(m(u, v)-\frac{1}{2} t\right)\right], \\
f^{\prime} f(u) \wedge v \text { if } m^{\prime}(f(u), v) \in\left[\frac{1}{2} t-2 \tau\left(m(u, v)-\frac{1}{2} t\right), \infty\right) .
\end{array}
\end{aligned}
$$

It follows in particular that $K_{t, 0}=\left.F_{t}^{\prime} \circ F_{t}\right|_{Y_{t}}$, if $m(u, v)=\frac{1}{2} t$, then $K_{t, \tau}(u \wedge v)=$ $F_{t}^{\prime} F_{t}(u \wedge v)$ and if $m(u, v) \geq \frac{3}{4} t$, then $K_{t, 1}(u \wedge v)=f^{\prime} f(u) \wedge v$. Let $D: U \times I \rightarrow U$, $(u, t) \mapsto D_{t}(u)$ be the homotopy relative to $s(Z)$ joining $f^{\prime} \circ f$ with the identity, i.e.

$$
D_{0}=f^{\prime} \circ f, \quad D_{1}=\mathrm{id}_{U} .
$$

For $t \in(0,1]$ and $\tau \in I$ define maps $H_{t, \tau}: U \wedge V \rightarrow U^{\prime} \wedge V$ by

$$
H_{t, \tau}(u \wedge v):= \begin{cases}F_{t}^{\prime} F_{t}(u \wedge v) & \text { if } m(u, v) \leq \frac{1}{2} t, \\ K_{t, 1}(u \wedge v) & \text { if } \frac{1}{2} \leq m(u, v) \leq \frac{3}{4} t, \\ D_{\frac{4 \tau}{t} m(u, v)-3 \tau}(u) \wedge v & \text { if } \frac{3}{4} \leq m(u, v) \leq t, \\ D_{\tau}(u) \wedge v & \text { if } t \leq m(u, v) .\end{cases}
$$

It follows that $F_{1}^{\prime} \circ F_{1} \simeq H_{1,0}$ rel $s_{\wedge}(Z \times \Omega)$ via the homotopy $\left\{\left.F_{1}^{\prime} \circ F_{1}\right|_{Y_{1}} \cup K_{1, t}\right\}_{t \in I}$ and $H_{1,0} \simeq H_{1,1}$ rel $s_{\wedge}(Z \times \Omega)$ via the homotopy $\left\{H_{1, t}\right\}_{t \in I}$. Define the final homotopy $J:(U \wedge V) \times I \ni(u \wedge v, t) \rightarrow J_{t}(u \wedge v) \in U^{\prime} \wedge V$ by

$$
J_{t}:= \begin{cases}\operatorname{id}_{U \wedge V} & \text { if } t=0 \\ H_{t, 1} & \text { if } t>0\end{cases}
$$


Observe, that $J$ is continuous in $\{(u \wedge v, 0): u \in s(Z)$ or $\sigma(\Omega)\}$ (hence continuous everywhere) and it is a homotopy relative to $s_{\wedge}(Z \times \Omega)$ joining $H_{1,1}$ with $\mathrm{id}_{U \wedge V}$. By composing the above homotopies we conclude that $F_{1}^{\prime} \circ F_{1}$ is homotopic to the identity in $\mathrm{Fib}_{Z \times \Omega}$. By the symmetric argument one can show that $F_{1} \circ F_{1}^{\prime} \simeq \mathrm{id}_{U^{\prime} \wedge V}$ in $\mathrm{Fib}_{Z \times \Omega}$, hence, by Proposition 3.3 , the proof is finished.

By Proposition 3.10 for fiberwise well-pointed spaces $\mathbf{U}$ over $Z$ and $\mathbf{U}^{\prime}$ over $Z^{\prime}$ we can define

$$
[\mathbf{U}]_{Z} \wedge\left[\mathbf{U}^{\prime}\right]_{Z^{\prime}}:=\left[\mathbf{U} \wedge \mathbf{U}^{\prime}\right]_{Z \times Z^{\prime}}
$$

The role of the unit element for smash product is played by the homotopy type $\Sigma^{0}$ (also denoted $\overline{1}$ ), i.e. the homotopy type of pointed 0 -dimensional sphere $\left(S^{0}, s_{0}\right)$ over $\left\{s_{0}\right\}$. Up to obvious identifications, for every fiberwise well-pointed space $\mathbf{U}$ over $Z$,

$$
[\mathbf{U}]_{Z} \wedge \Sigma^{0}=\Sigma^{0} \wedge[\mathbf{U}]_{Z}=[\mathbf{U}]_{Z}
$$

\section{FibERWISE POINTED SPACES GENERATED BY CLOSED PAIRS}

Let $X$ be a topological space and let $\omega: X \rightarrow Z$ be a continuous map. For $P=\left(P_{1}, P_{2}\right)$ a pair of closed spaces in $X$ (i.e. $\left.P_{2} \subset P_{1} \subset X\right)$, we define $U_{\omega}(P)$ as the adjunction $P_{1} \cup_{\left.\omega\right|_{P_{2}}} Z$ (see [E]), i.e.

$$
U_{\omega}(P):=Z \times 0 \cup P_{1} \times 1 / \sim,
$$

where $\sim$ denotes the minimal equivalence relation such that $(x, 1) \sim(\omega(x), 0)$ for every $x \in P_{2}$.

The equivalence class of an element $(y, i) \in Z \times 0 \cup P_{1} \times 1$ with respect to $\sim$ will be denoted by $[y, i]_{\omega, P}$ or briefly $[y, i]_{P}$. We define the quotient map by

$$
q_{\omega, P}: Z \times 0 \cup P_{1} \times 1 \ni(y, i) \rightarrow[y, i]_{\omega, P} \in U_{\omega}(P) .
$$

Proposition 4.1. If $\left.\omega\right|_{P_{2}}$ is a closed map, then the quotient map $q_{\omega, P}$ is also closed.

Define $r_{\omega, P}: U_{\omega}(P) \rightarrow Z$ and $s_{\omega, P}: Z \rightarrow U_{\omega}(P)$ by

$$
\begin{gathered}
r_{\omega, P}\left([x, 1]_{\omega, P}\right):=\omega(x), r_{\omega, P}\left([z, 0]_{\omega, P}\right):=z, \\
s_{\omega, P}(z):=[z, 0]_{\omega, P} .
\end{gathered}
$$

It is easy to verify that the maps $r_{\omega, P}, s_{\omega, P}$ are well defined and continuous and the triple $\mathbf{U}_{\omega}(P):=\mathbf{U}(P):=\left(U_{\omega}(P), r_{\omega, P}, s_{\omega, P}\right)$ is a fiberwise pointed space over $Z$.

The following result is a straightforward consequence of definitions and facts presented at the end of Section [3]

Proposition 4.2. If $P$ is a cofibered closed pair, then $\mathbf{U}_{\omega}(P)$ is a fiberwise wellpointed space over $Z$.

If $P \subset Q$ are closed pairs in $X$, then the inclusion induces a fiberwise pointed map (hence f.d. map, i.e. a morphism in $\mathrm{Fib}_{Z}$ ):

$$
\mathbf{U}_{\omega}\left(\iota_{P Q}\right): \mathbf{U}_{\omega}(P) \rightarrow \mathbf{U}_{\omega}(Q), \quad[u, i]_{P} \mapsto[u, i]_{Q}
$$

Proposition 4.3. If $P \subset Q$ are closed pairs such that $P_{1} \backslash P_{2}=Q_{1} \backslash Q_{2}$ and $\left.\omega\right|_{Q_{2}}$ is closed, then $\mathbf{U}_{\omega}\left(\iota_{P Q}\right)$ is an isomorphism in $\mathrm{Fib}_{Z}$. 
Proof. In order to prove the result it suffices to show that $\mathbf{U}_{\omega}\left(\iota_{P Q}\right)$ is a homomorphism. Since $\mathbf{U}_{\omega}\left(\iota_{P Q}\right)$ is a continuous bijective map, we only need to prove that $\mathbf{U}_{\omega}\left(\iota_{P Q}\right) \circ q_{\omega, P}$ is closed. Actually, the latter map is the composition of the inclusion $Z \times 0 \cup P_{1} \times 1 \hookrightarrow Z \times 0 \cup Q_{1} \times 1$ and $q_{\omega, Q}$. Since $P_{1}$ is closed, the inclusion is a closed map, and thus the assertion follows by Proposition 4.1 .

Now we present examples and results concerning fiberwise deforming homotopy type of the spaces introduced in this section. We begin with a trivial observation that for every map $\omega: X \rightarrow Z$,

$$
\left[\mathbf{U}_{\omega}(\varnothing)\right]_{Z}=\overline{0}_{Z}
$$

More interesting fiberwise deforming homotopy types are described in the following:

Example 4.4. Let $C_{1}=S^{1}$ and $C_{2}=\{x:|x-(2,0)|=1\}$ be two circles in the plane. Consider four closed pairs in $\mathrm{R}^{2} \backslash 0$ :

$$
P_{1}=\left(C_{1}, \varnothing\right), P_{2}=\left(C_{2}, \varnothing\right), P_{3}=\left(C_{1},\{(1,0)\}\right), P_{4}=\left(C_{2},\{(3,0)\}\right) .
$$

Putting $\omega=\operatorname{id}_{\mathrm{R}^{2} \backslash 0}$, we get $\left[\mathbf{U}\left(P_{i}\right)\right]_{\mathrm{R}^{2} \backslash 0} \neq\left[\mathbf{U}\left(P_{3}\right)\right]_{\mathrm{R}^{2} \backslash 0}$ for $i=1,2$, because $P_{3}$ is connected. By Proposition 3.5, we also have $\left[\mathbf{U}\left(P_{1}\right)\right]_{\mathrm{R}^{2} \backslash 0} \neq\left[\mathbf{U}\left(P_{2}\right)\right]_{\mathrm{R}^{2} \backslash 0}$. However, one can show that $\left[\mathbf{U}\left(P_{3}\right)\right]_{\mathrm{R}^{2} \backslash 0}=\left[\mathbf{U}\left(P_{4}\right)\right]_{\mathrm{R}^{2} \backslash 0}$.

The following example shows that for a given pair $P$ the fiberwise deforming homotopy types of $\mathbf{U}_{\omega}(P)$ can be distinct for various maps $\omega$ from the same homotopy class.

Example 4.5. Let $X=Z=\mathrm{R}$ and let $P=\left(P_{1}, P_{2}\right)$ be given by

$$
P_{1}=\left\{\frac{1}{n}: n \in \mathrm{Z}^{+}\right\} \cup\{0\}, \quad P_{2}=\{0\} .
$$

Denote by $\tau_{1}$ the translation $x \mapsto x+1$. Then $\tau_{1} \simeq \operatorname{id}_{\mathcal{R}}$, but

$$
\left[\mathbf{U}_{\tau_{1}}(P)\right]_{\mathrm{R}} \neq\left[\mathbf{U}_{\mathrm{id}_{\mathrm{R}}}(P)\right]_{\mathrm{R}} .
$$

This can be shown by essentially the same argument as in Example 3.8

In the next proposition we indicate that $\left[\mathbf{U}_{\omega}(P)\right]_{Z}$ depends only on the homotopy class of $\omega$ if $P$ is cofibered. Let $\phi, \psi: X \rightarrow Z$ be continuous maps.

Proposition 4.6. If $P=\left(P_{1}, P_{2}\right)$ is a cofibered closed pair and $\left.\left.\phi\right|_{P_{2}} \simeq \psi\right|_{P_{2}}: P_{2} \rightarrow$ $Z$, then

$$
\left[\mathbf{U}_{\phi}(P)\right]_{Z}=\left[\mathbf{U}_{\psi}(P)\right]_{Z} .
$$

Proof. Let $(G, a)$ be a Strøm structure for $P$ and let $\omega_{t}: P_{2} \rightarrow Z, t \in I$, be a homotopy joining $\omega_{0}=\left.\phi\right|_{P_{2}}$ with $\omega_{1}=\left.\psi\right|_{P_{2}}$. Define maps $f: \mathbf{U}_{\omega_{0}}(P) \rightarrow \mathbf{U}_{\omega_{1}}(P)$ and $f^{\prime}: \mathbf{U}_{\omega_{1}}(P) \rightarrow \mathbf{U}_{\omega_{0}}(P)$ by

$$
\begin{aligned}
& f\left([z, 0]_{\omega_{0}, P}\right):=[z, 0]_{\omega_{1}, P}, \\
& f\left([x, 1]_{\omega_{0}, P}\right):= \begin{cases}{\left[\omega_{2 a(x)} G_{1}(x), 0\right]_{\omega_{1}, P}} & \text { if } x \in \operatorname{cl} a^{-1}\left(\left[0, \frac{1}{2}\right)\right), \\
{\left[G_{2-2 a(x)}(x), 1\right]_{\omega_{1}, P}} & \text { if } \frac{1}{2} \leq a(x),\end{cases} \\
& f^{\prime}\left([z, 0]_{\omega_{1}, P}\right):=[z, 0]_{\omega_{0}, P},
\end{aligned}
$$


Both the maps $f$ and $f^{\prime}$ are f.d. as one can directly check. Define a homotopy $h: U_{\omega_{0}}(P) \times I \rightarrow U_{\omega_{0}}(P)$ by

$$
\begin{gathered}
h\left([z, 0]_{\omega_{0}, P}, t\right):=[z, 0]_{\omega_{0}, P}, \\
\qquad \begin{cases}{\left[\omega_{2 t a(x)} G_{1}(x), 0\right]_{\omega_{0}, P}} & \text { if } x \in \operatorname{cl} a^{-1}\left(\left[0, \frac{1}{2}\right]\right), \\
{\left[\omega_{t\left(1-2 a\left(G_{2-2 a(x)}(x)\right)\right.} G_{1} G_{2-2 a(x)}, 0\right]_{\omega_{0}, P},} & \text { if } \frac{1}{2} \leq a(x), \\
{\left[G_{2-2 a\left(G_{2-2 a(x)}(x)\right)} G_{2-2 a(x)}(x), 1\right]_{\omega_{0}, P}} & \text { if } \frac{1}{2} \leq a(x), \\
& \frac{1}{2} \leq a G_{2-2 a(x)}(x) .\end{cases}
\end{gathered}
$$

Thus $f^{\prime} \circ f \simeq h_{0} \operatorname{rel} s_{\omega_{0}, P}(Z)$. Define another homotopy $j: U_{\omega_{0}}(P) \times I \rightarrow U_{\omega_{0}}(P)$ by

$$
j\left([z, 0]_{\omega_{0}, P}, t\right):=[z, 0]_{\omega_{0}, P}
$$

$$
\begin{aligned}
& j\left([x, 1]_{\omega_{0}, P}, t\right):= \\
& \begin{cases}{\left[\omega_{0} G_{1}(x), 0\right]_{\omega_{0}, P}} & \text { if } x \in \operatorname{cl} a^{-1}\left(\left[0, \frac{1}{2}\right)\right), \\
{\left[\omega_{0} G_{1} G_{2-2 a(x)}(x), 0\right]_{\omega_{0}, P}} & \text { if } \frac{1}{2} \leq a(x), \\
{\left[G_{t+(1-t)\left(2-2 a\left(G_{2-2 a(x)}(x)\right)\right)} G_{2-2 a(x)}(x), 1\right]_{\omega_{0}, P}} & \text { if } \frac{1}{2} \leq a(x), \\
& \frac{1}{2} \leq a G_{2-2 a(x)}(x),\end{cases}
\end{aligned}
$$

hence $h_{0} \simeq j_{1} \operatorname{rel} s_{\omega_{0}, P}(Z)$. The next homotopy $k: U_{\omega_{0}}(P) \times I \rightarrow U_{\omega_{0}}(P)$ is given by

$$
\begin{gathered}
k\left([z, 0]_{\omega_{0}, P}, t\right):=[z, 0]_{\omega_{0}, P}, \\
k\left([x, 1]_{\omega_{0}, P}, t\right):= \\
\begin{cases}{\left[\omega_{0} G_{1} G_{t}(x), 0\right]_{\omega_{0}, P}} & \text { if } a(x) \leq \frac{1}{2}, \\
{\left[\omega_{0} G_{1} G_{t(2-2 a(x))}(x), 0\right]_{\omega_{0}, P}} & \text { if } \frac{1}{2} \leq a(x), a G_{t(2-2 a(x))}(x) \leq \frac{1}{2}, \\
{\left[G_{1} G_{t(2-2 a(x))}(x), 1\right]_{\omega_{0}, P}} & \text { if } \frac{1}{2} \leq a(x), \frac{1}{2} \leq a G_{2-2 a(x)}(x) .\end{cases}
\end{gathered}
$$

Since $G$ comes from the Strøm structure, $G_{1} G_{1}(x)=G_{1}(x)$ if $a(x)<1$, hence $k_{1}=j_{1}$ and thus $j_{1} \simeq k_{0} \operatorname{rel} s_{\omega_{0}, P}(Z)$. The last homotopy $m: U_{\omega_{0}}(P) \times I \rightarrow U_{\omega_{0}}(P)$ is simply defined by $m_{t}\left([z, 0]_{\omega_{0}, P}\right)=[z, 0]_{\omega_{0}, P}, m_{t}\left([x, 1]_{\omega_{0}, P}\right)=\left[G_{t}(x), 1\right]_{\omega_{0}, P}$. It is easy to check that $k_{0}=m_{1}$, hence $k_{0} \simeq \operatorname{id}_{U_{\omega_{0}}(P)} \operatorname{rel} s_{\omega_{0}, P}(Z)$. By composing the above homotopies we conclude that $f^{\prime} \circ f \simeq \operatorname{id}_{U_{\omega_{1}}(P)}$ rel $s_{\omega_{0}, P}(Z)$. Similarly, one can prove that also $f \circ f^{\prime}$ is homotopic to the identity, hence by Proposition 3.3 the proof is finished.

Let $z_{0}$ be a point in $Z$ and let $c:=c_{z_{0}}: X \rightarrow Z, x \mapsto z_{0}$, be a constant map. If $P$ is a closed pair, then $U_{c}(P)=P_{1} / P_{2} \vee\left(Z, z_{0}\right)$ and $r_{c, P}\left([x, 1]_{c, P}\right)=z_{0}$ for every $x \in P_{1}$. In that case the fiberwise deforming homotopy type $\left[\mathbf{U}_{c}(P)\right]_{Z}$ is called simple.

Corollary 4.7. If $P=\left(P_{1}, P_{2}\right)$ is cofibered and $P_{1}$ is contractible in $X$, then $\left[\mathbf{U}_{\omega}(P)\right]_{Z}$ is simple for every $\omega: X \rightarrow Z$. 
Let $P$ be a closed pair in $X$ and let $\zeta: Z \rightarrow Z^{\prime}$ be a continuous map. Define $\zeta_{P}: U_{\omega}(P) \rightarrow U_{\zeta \circ \omega}(P)$ by

$$
\zeta_{P}\left([z, 0]_{\omega, P}\right)=[\zeta(z), 0]_{\zeta \circ \omega, P}, \quad \zeta_{P}\left([x, 1]_{\omega, P}\right)=[x, 1]_{\zeta \circ \omega, P} .
$$

Since $\zeta_{P} \circ q_{\omega, P}=q_{\zeta \circ \omega, P} \circ\left(\zeta \times 0 \cup \operatorname{id}_{P_{1} \times 1}\right)$, the map $\zeta_{P}$ is continuous. Assume that $Q$ is another closed pair and let $f: \mathbf{U}_{\omega}(P) \rightarrow \mathbf{U}_{\omega}(Q)$ be an f.d. map. Define $f^{\prime}: U_{\zeta \circ \omega}(P) \rightarrow U_{\zeta \circ \omega}(Q)$ by

$$
f^{\prime}\left(\left[z^{\prime}, 0\right]_{\zeta \circ \omega, P}\right)=\left[z^{\prime}, 0\right]_{\zeta \circ \omega, Q}, \quad f^{\prime}\left([x, 1]_{\zeta \circ \omega, P}\right)=\left(\zeta_{Q} \circ f\right)\left([x, 1]_{\omega, P}\right) .
$$

The correctness of the definition of $f^{\prime}$ is a consequence of the fact that $f\left([z, 0]_{\omega, P}\right)=$ $[z, 0]_{\omega, Q}$ for every $z \in Z$ (recall that $f$ is f.d.). The map $f^{\prime}$ is continuous because the restrictions $\left.\left(f^{\prime} \circ q_{\zeta \circ \omega, P}\right)\right|_{Z^{\prime} \times 0}$ and $\left.\left(f^{\prime} \circ q_{\zeta \circ \omega, P}\right)\right|_{P_{1} \times 1}$ are continuous, which follows from the description of $f^{\prime}$. Moreover, $f^{\prime}$ is also f.d. Indeed, let $h: U_{\omega}(P) \times I \rightarrow Z$ be a homotopy relative to $s_{\omega, P}(Z)$ joining $r_{\omega, Q} \circ f$ with $r_{\omega, P}$. Define $h^{\prime}: U_{\zeta \circ \omega}(P) \times I \rightarrow$ $Z^{\prime}$ by

$$
h^{\prime}\left(\left[z^{\prime}, 0\right]_{\zeta \circ \omega, P}, t\right)=z^{\prime}, \quad h^{\prime}\left([x, 1]_{\zeta \circ \omega, P}, t\right)=(\zeta \circ h)\left([x, 1]_{\omega, P}, t\right) .
$$

It follows by Remark 3.9 and an argument similar to the one above that $h^{\prime}$ is correctly defined and continuous, and is the required homotopy joining $r_{\zeta \circ \omega, Q} \circ f$ with $r_{\zeta \circ \omega, P}$, hence $f^{\prime}: \mathbf{U}_{\zeta \circ \omega}(P) \rightarrow \mathbf{U}_{\zeta \circ \omega}(Q)$ is f.d. By a suitable modification of the above arguments one can show that if $f \simeq g: \mathbf{U}_{\omega}(P) \rightarrow \mathbf{U}_{\omega(Q)}$ in $\mathrm{Fib}_{Z}$, then also the corresponding f.d. maps $f^{\prime}, g^{\prime}: \mathbf{U}_{\zeta \circ \omega}(P) \rightarrow \mathbf{U}_{\zeta \circ \omega}(Q)$ are homotopic in $\mathrm{Fib}_{Z^{\prime}}$. As a conclusion we get the following:

Proposition 4.8. If $\left[\mathbf{U}_{\omega}(P)\right]_{Z}=\left[\mathbf{U}_{\omega(Q)}\right]_{Z}$ and $\zeta: Z \rightarrow Z^{\prime}$ is continuous, then $\left[\mathbf{U}_{\zeta \circ \omega}(P)\right]_{Z^{\prime}}=\left[\mathbf{U}_{\zeta \circ \omega}(Q)\right]_{Z^{\prime}}$.

As immediate corollaries from the above proposition we get results which assert that the information provided by $\left[\mathbf{U}_{\omega}(\cdot)\right]_{Z}$ is maximal in the case $\omega=\mathrm{id}_{X}$ and minimal if $\omega$ is a constant map onto a one-point space.

Corollary 4.9. If $\left[\mathbf{U}_{\omega}(P)\right]_{Z}=\left[\mathbf{U}_{\omega}(Q)\right]_{Z}$ for some $\omega: X \rightarrow Z$, then $\left[P_{1} / P_{2}\right]=$ $\left[Q_{1} / Q_{2}\right]$.

Corollary 4.10. If $\left[\mathbf{U}_{\mathrm{id}_{X}}(P)\right]_{X}=\left[\mathbf{U}_{\mathrm{id}_{X}}(Q)\right]_{X}$, then for every space $Z$ and every continuous map $\omega: X \rightarrow Z,\left[\mathbf{U}_{\omega}(P)\right]_{Z}=\left[\mathbf{U}_{\omega}(Q)\right]_{Z}$.

Propositions 4.6 and 4.8 imply

Corollary 4.11. If $P$ and $Q$ are cofibered pairs, and $\zeta: Z \rightarrow Z^{\prime}$ is a homotopy equivalence, then $\left[\mathbf{U}_{\omega}(P)\right]_{Z}=\left[\mathbf{U}_{\omega}(Q)\right]_{Z}$ if and only if $\left[\mathbf{U}_{\zeta \circ \omega}(P)\right]_{Z^{\prime}}=\left[\mathbf{U}_{\zeta \circ \omega}(Q)\right]_{Z^{\prime}}$.

We conclude this section by results on sums and products of closed pairs. Let $P=\left(P_{1}, P_{2}\right)$ and $Q=\left(Q_{1}, Q_{2}\right)$ be disjoint closed pairs in $X$, i.e. $P_{1} \cap Q_{1}=\varnothing$. Then

$$
\mathbf{U}_{\omega}(P \cup Q)=\mathbf{U}_{\omega}(P) \vee \mathbf{U}_{\omega(Q)},
$$

hence

Proposition 4.12. If $P$ and $Q$ are disjoint closed pairs in $X$, then

$$
\left[\mathbf{U}_{\omega}(P \cup Q)\right]_{Z}=\left[\mathbf{U}_{\omega}(P)\right]_{Z} \vee\left[\mathbf{U}_{\omega}(Q)\right]_{Z}
$$


Now let $P$ be a closed pair in $X$ and $P^{\prime}$ in $X^{\prime}$, and let $\omega: X \rightarrow Z$ and $\omega^{\prime}: X^{\prime} \rightarrow$ $Z^{\prime}$ be continuous. Recall that $P \times P^{\prime}=\left(P_{1} \times P_{1}^{\prime}, P_{1} \times P_{2}^{\prime} \cup P_{2} \times P_{1}^{\prime}\right)$. Then, up to a natural isomorphism,

$$
\mathbf{U}_{\omega \times \omega^{\prime}}\left(P \times P^{\prime}\right)=\mathbf{U}_{\omega}(P) \wedge \mathbf{U}_{\omega^{\prime}}\left(P^{\prime}\right) .
$$

As a consequence of Proposition 4.2 we get

Proposition 4.13. If $P$ and $P^{\prime}$ are cofibered closed pairs, then

$$
\left[\mathbf{U}_{\omega \times \omega^{\prime}}\left(P \times P^{\prime}\right)\right]_{Z \times Z^{\prime}}=\left[\mathbf{U}_{\omega}(P)\right]_{Z} \wedge\left[\mathbf{U}_{\omega^{\prime}}\left(P^{\prime}\right)\right]_{Z^{\prime}}
$$

\section{REGULAR INDEX PAIRS AND ISOLATING BLOCKS}

Let $X$ be a metrizable locally compact space and let the map $\varphi: X \times \mathrm{R}^{+} \ni$ $(x \cdot t) \rightarrow x \cdot t \in X$ be a semiflow. We recall several concepts from the theory of isolated invariant sets; cf. [Co, $\mathrm{Ch}],[\mathrm{Ry}]$, and $[\mathrm{Sm}]$.

Let $I$ be an interval in $\mathrm{R}$ with $0 \in I$ and let $A \subset X$. A single valued mapping $\sigma: I \rightarrow A$ is called a solution for $\varphi$ through $x \in A$ if $\sigma(t+s)=\varphi(\sigma(t), s)$ for all $t, t+s \in I, s>0$, and $\sigma(0)=x$.

Given an arbitrary set $A \subset X$, we define the maximal invariant part of $A$ by

$$
\text { Inv } A:=\{x \in A: \exists \sigma: \mathrm{R} \rightarrow A \text { a solution for } \varphi \text { through } x\}
$$

the exit function on $A$ by

$$
\varepsilon_{A}: A \ni x \rightarrow \sup \{t \geq 0 \mid x \cdot[0, t] \subset A\} \in[0, \infty]
$$

and the exit set $A^{-}:=\varepsilon_{A}^{-1}(0)$.

A set $S \subset X$ is called an isolated invariant set if $S$ is compact and there exists $U$, a neighborhood of $S$, such that $S=\operatorname{Inv} U$. A compact subset $N \subset X$ is called an isolating neighborhood for $\varphi$ if $\operatorname{Inv} N \subset \operatorname{int} N$. It is easy to check that if $N$ is an isolating neighborhood, then $\operatorname{Inv} N$ is an isolated invariant set. An isolating neighborhood $B$ is called an isolating block iff $B^{-}$is closed. The following result is a variant of Ważewski's theorem ([Co, Sec. II.2]):

Proposition 5.1 (Ważewski). An isolating neighborhood $B$ is an isolating block if and only if the map $\varepsilon_{B}$ is continuous.

Let $N$ be an isolating neighborhood for $\varphi$. A pair $P=\left(P_{1}, P_{2}\right)$ of compact subsets $P_{2} \subset P_{1} \subset N$ is called an index pair in $N$ if the following conditions are satisfied:

$$
\begin{gathered}
x \in P_{i}, t>0, x \cdot[0, t] \subset N \Rightarrow x \cdot[0, t] \subset P_{i} \quad(i=1,2), \\
x \in P_{1}, t>0, x \cdot t \notin N \Rightarrow \exists t^{\prime} \in[0, t] \text { with } x \cdot t^{\prime} \in P_{2}, x \cdot\left[0, t^{\prime}\right] \subset N,
\end{gathered}
$$

$$
\operatorname{Inv} N \subset \operatorname{int}\left(P_{1} \backslash P_{2}\right) \text {. }
$$

For an index pair $P$ we define functions $\sigma_{P}, \tau_{P}: P_{1} \rightarrow[0, \infty]$ by

$$
\begin{aligned}
\sigma_{P}(x) & := \begin{cases}\varepsilon_{\operatorname{cl}\left(P_{1} \backslash P_{2}\right)}(x) & \text { if } x \in \operatorname{cl}\left(P_{1} \backslash P_{2}\right), \\
0 & \text { if } x \in \operatorname{int}_{P_{1}} P_{2},\end{cases} \\
\tau_{P}(x): & = \begin{cases}\sup \left\{t \geq 0 \mid x \cdot[0, t] \subset P_{1} \backslash P_{2}\right\} & \text { if } x \in P_{1} \backslash P_{2}, \\
0 & \text { if } x \in P_{2} .\end{cases}
\end{aligned}
$$

Proposition 5.2. $\sigma_{P}$ is upper semicontinuous, $\tau_{P}$ is lower semicontinuous and $\tau_{P}(x) \leq \sigma_{P}(x)$ for $x \in P_{1}$. 


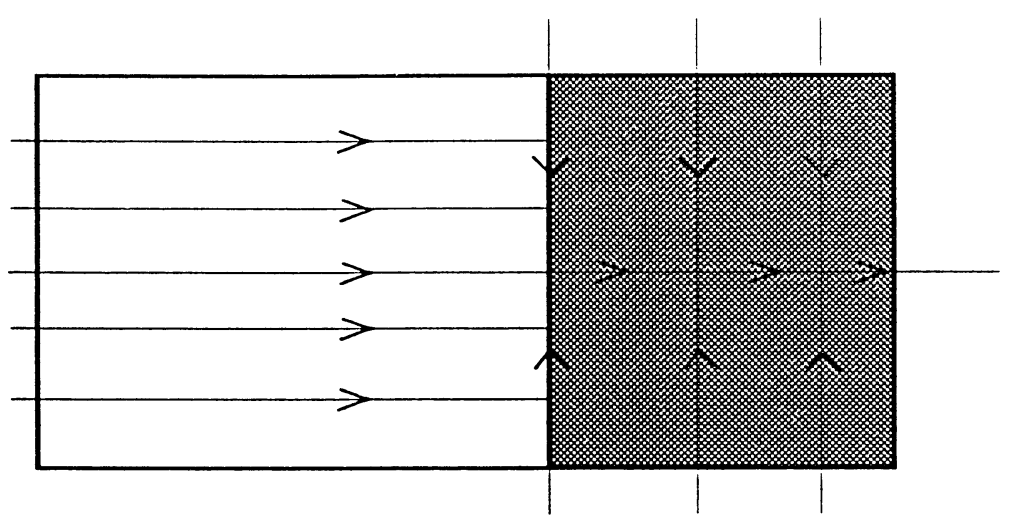

Figure 2. An index pair for a semiflow with $\sigma_{P} \neq \tau_{P}$

One easily verifies the following proposition.

Proposition 5.3. If $\varphi$ is a flow, then the following three conditions are equivalent.

1. $\sigma_{P}$ is continuous.

2. $\tau_{P}$ is continuous.

3. $\sigma_{P}=\tau_{P}$.

This proposition is not true for semiflows as the example in Figure 2 illustrates. The large rectangle is $P_{1}$, and the shaded area is $P_{2}$.

We call the index pair $P$ regular if $\tau_{P}(x)=\sigma_{P}(x)$ for $x \in P_{1}$.

Proposition 5.4. If $P$ is a regular index pair, then $\operatorname{cl}\left(P_{1} \backslash P_{2}\right)$ is an isolating block.

Proof. Since $\sigma_{P}=\tau_{P}$, by Proposition 5.2 they are both continuous. In particular, $\varepsilon_{\mathrm{cl}\left(P_{1} \backslash P_{2}\right)}=\left.\sigma_{P}\right|_{\mathrm{cl}\left(P_{1} \backslash P_{2}\right)}$ is continuous and therefore the exit set $\left(\operatorname{cl}\left(P_{1} \backslash P_{2}\right)\right)^{-}$is closed. It follows that $\mathrm{cl}\left(P_{1} \backslash P_{2}\right)$ is an isolating block.

A convenient way of constructing regular index pairs or isolating blocks is by means of Lyapunov functions. A function $\alpha: A \rightarrow[0, \infty]$ is called strictly increasing (decreasing) along the trajectories of $\varphi$ if for any $x \in A$ and $t>0$ such that $x \cdot[0, t] \subset A$ and $\alpha(x)>0$ there is $\alpha(x \cdot t)>\alpha(x)(\alpha(x \cdot t)<\alpha(x))$. A pair of functions $\mu=\left(\mu_{1}, \mu_{2}\right)$, where $\mu_{i}: N \rightarrow[0, \infty]$ for $i=1,2$ will be called a Lyapunov pair in $N$ if $\mu_{1}$ is strictly decreasing, $\mu_{2}$ is strictly increasing along the trajectories of $\varphi$ and Inv $N=\mu_{1}^{-1}(0) \cap \mu_{2}^{-1}(0)$. The following theorem is a by-product of the proof of Theorem 1.5.1 in Ry.

Theorem 5.5. If $N$ is an isolating neighborhood for $S$ which is sufficiently close to $S$, then it admits a Lyapunov pair.

A standard compactness argument leads to the following:

Proposition 5.6. Assume $U \subset N$ is an open neighborhood of $S=\operatorname{Inv} N$ and $\mu$ is a Lyapunov pair in $N$. Then there exists an $\varepsilon>0$ such that

$$
\mu_{1}^{-1}([0, \varepsilon]) \cap \mu_{2}^{-1}([0, \varepsilon]) \subset U .
$$

Lyapunov pairs may be used to construct regular index pairs. Let $N$ be an isolating neighborhood which admits a Lyapunov pair $\mu$. Let $U \subset \operatorname{int} N$ be open 
and let $\varepsilon>0$ be such that (5.6) is satisfied. Put $\alpha_{1}, \alpha_{2} \in(0, \varepsilon)$ and for $\alpha:=\left(\alpha_{1}, \alpha_{2}\right)$ define

$$
P^{\alpha}:=\left(P_{1}^{\alpha}, P_{2}^{\alpha}\right)
$$

where

$$
\begin{aligned}
& P_{1}^{\alpha}:=\left\{x \in N \mid \mu_{1}(x) \leq \alpha_{1}\right\} \\
& P_{2}^{\alpha}:=\left\{x \in P_{1}^{\alpha} \mid \mu_{2}(x) \geq \alpha_{2}\right\}
\end{aligned}
$$

We have the following:

Theorem 5.7. $P^{\alpha}$ is a regular index pair in $N$ which satisfies $P_{1}^{\alpha} \backslash P_{2}^{\alpha} \subset U$.

Proof. Property (5.1) is obvious. To see (5.2) take $x \in P_{1}^{\alpha}$ and $t>0$ such that $x \cdot t \notin N$. Put $t^{\prime}:=\sigma_{N}(x)$. Then $x \cdot t^{\prime} \in$ bd $N$, i.e. $\mu_{2}\left(x \cdot t^{\prime}\right) \geq \varepsilon \geq \alpha_{2}$. This proves (5.2). Property (5.3) is obvious. It remains to show that $P^{\alpha}$ is regular. We have

$$
P_{1}^{\alpha} \backslash P_{2}^{\alpha}=\left\{x \in N \mid \mu_{1}(x) \leq \alpha_{1}, \mu_{2}(x)<\alpha_{2}\right\}
$$

and

$$
\operatorname{cl}\left(P_{1}^{\alpha} \backslash P_{2}^{\alpha}\right) \subset\left\{x \in N \mid \mu_{1}(x) \leq \alpha_{1}, \mu_{2}(x) \leq \alpha_{2}\right\} .
$$

This if $x \in P_{1}$, we get $\mu_{2}\left(x \cdot \tau_{P}(x)\right)=\alpha_{2}$ and $\mu_{2}\left(x \cdot \sigma_{P}(x)\right) \leq \alpha_{2}$. Since $\mu_{2}$ is increasing along solutions, it must be $\sigma_{P}(x) \leq \tau_{P}(x)$. The other inequality follows from Proposition 5.2

As a consequence we obtain the following result.

Lemma 5.8. Assume $N$ is an isolating neighborhood which admits a Lyapunov pair. Then for any $n \in \mathrm{Z}^{+}, n \geq 2$, there exists a sequence of regular index pairs $P^{1}, P^{2}, \ldots, P^{n}$ in $N$ such that $P^{i-1} \subset \operatorname{int} P^{i}$ for $i=2,3, \ldots, n$.

Proof. Choose numbers $0<\alpha_{1}^{1}<\alpha_{1}^{2}<\cdots<\alpha_{1}^{n}<\varepsilon, \varepsilon>\alpha_{2}^{1}>\alpha_{2}^{2}>\cdots \alpha_{2}^{n}>0$ and put $P^{i}:=P^{\alpha^{i}}$, where $\alpha^{i}:=\left(\alpha_{1}^{i}, \alpha_{2}^{i}\right)$. It is now straightforward to verify that the assertion is satisfied.

\section{Proposition 5.9.}

(i) If $P$ and $Q$ are regular index pairs in $N$, then so is $P \cap Q:=\left(P_{1} \cap Q_{1}, P_{2} \cap Q_{2}\right)$.

(ii) If $P \subseteq Q$ are regular index pairs for $N$, then so are $Q^{\prime}:=\left(P_{1}, P_{1} \cap Q_{2}\right)$ and $Q^{\prime \prime}:=\left(P_{1} \cup Q_{2}, Q_{2}\right)$.

Proof. It is an easy exercise to show that $P \cap Q, Q^{\prime}, Q^{\prime \prime}$ satisfy properties ([5.1) (5.3)). To show regularity of $P \cap Q, Q^{\prime}, Q^{\prime \prime}$ observe first that it follows easily from (5.5) that

$$
P \subset Q \Rightarrow \tau_{P} \geq \tau_{Q \mid P_{1}}
$$

We will show that

$$
\tau_{P \cap Q}=\max \left(\tau_{P \mid P_{1} \cap Q_{1}}, \tau_{Q \mid P_{1} \cap Q_{1}}\right) .
$$

One inequality follows immediately from (5.9). Assume the other is not true. Then there exists an $x \in P_{1} \cap Q_{1}$ and a $t \in \mathrm{R}^{+}$such that

$$
\tau_{P \cap Q}(x)>t>\max \left(\tau_{P}(x), \tau_{Q}(x)\right) .
$$

In particular, we get from (15.5) that

$$
x \cdot[0, t] \cap P_{2} \cap Q_{2}=\varnothing
$$


and for some $t_{1}, t_{2} \in[0, t]$

$$
x \cdot t_{1} \in P_{2}, \quad x \cdot t_{2} \in Q_{2} .
$$

It follows from (5.1) that $x \cdot \max \left(t_{1}, t_{2}\right) \in P_{2} \cap Q_{2}$, a contradiction. Thus $\tau_{P \cap Q}$ is continuous as a maximum of two continuous functions and consequently $P \cap Q$ is a regular index pair.

A similar argument shows that $\tau_{Q^{\prime}}=\tau_{Q \mid P_{1}}$ and $\tau_{Q^{\prime \prime}}=\tau_{Q \mid P_{1} \cup Q_{2}}$ and the conclusion follows.

\section{The Conley Index over A BASE}

In the remainder of this paper we assume that $\omega: X \rightarrow Z$ is continuous and $Z$ is a Hausdorff space. We connect results on semiflows from the previous section with the topological background from Section 4. First observe that a regular index pair is cofibered. This is an immediate consequence of Proposition 5.4 and $[\mathrm{Ry}, \mathrm{Th}$. 3.7]. Actually, it can be easily verified by constructing a Strøm structure using the exit function; similar constructions will frequently appear in the remainder of this paper. As a consequence of Proposition 4.2, we have

Proposition 6.1. If $P$ is a regular index pair, then $\mathbf{U}_{\omega}(P)$ is a fiberwise wellpointed space over $Z$.

We will prove the following two theorems. The second clearly implies Theorem 2.1 .

Theorem 6.2. Let $N$ be an isolating neighborhood and let $P \subset Q$ be two regular index pairs in $N$. Then $\mathbf{U}_{\omega}\left(\iota_{P Q}\right)$ is a fiberwise deforming homotopy equivalence.

Theorem 6.3. Let $S$ be an isolated invariant set. Then $h_{\omega}(S, \varphi):=\left[\mathbf{U}_{\omega}(P)\right]_{Z}$ is independent of the choice of an isolating neighborhood $N$ of $S$ and of a regular index pair $P$ in $N$.

The fiberwise deforming homotopy type $h_{\omega}(S, \varphi)$ given by the above theorem (denoted shortly by $h_{\omega}(S)$ if $\varphi$ is clear from the context) will be called the Conley index of $S$ over base $Z$ with base map $\omega$ or just the Conley index of $S$ over $Z$ if $\omega$ is clear from context.

Proof of Theorem 6.2. The proof proceeds in two steps.

Step 1. We make an extra assumption that $P$ and $Q$ differ by at most one coordinate, i.e. $P_{1}=Q_{1}$ or $P_{2}=Q_{2}$. Let us assume first that $P_{1}=Q_{1}$. Fix an $\varepsilon>$ 0 . For $x \in Q_{1}$ put $\alpha(x):=\sigma_{P}(x)-\sigma_{Q}(x)$ and define the map $f: \mathbf{U}_{\omega}(Q) \rightarrow \mathbf{U}_{\omega}(P)$ by

$$
\begin{aligned}
f\left([x, i]_{Q}\right):= & \begin{cases}{\left[\omega\left(x \cdot\left(\sigma_{Q}(x)+\frac{\sigma_{Q}(x)}{\varepsilon} \alpha(x)\right), 0\right]_{P}\right.} & \text { if } i=1,0 \leq \sigma_{Q}(x) \leq \varepsilon \\
{\left[x \cdot \frac{2 \varepsilon-\sigma_{Q}(x)}{\varepsilon}(\varepsilon+\alpha(x)), 1\right]_{P}} & \text { if } i=1, \varepsilon \leq \sigma_{Q}(x) \leq 2 \varepsilon, \\
{[x, 1]_{P}} & \text { if } i=1, \sigma_{Q}(x) \geq 2 \varepsilon \\
{[x, 0]_{P}} & \text { if } i=0 .\end{cases}
\end{aligned}
$$

It is a lengthy but straightforward task to verify that the map is well defined and continuous. We will show that $f$ is a homotopy inverse of $\mathbf{U}_{\omega(\iota P Q)}$. To this end 
consider the homotopy $F_{\lambda}: \mathbf{U}_{\omega}(Q) \rightarrow \mathbf{U}_{\omega}(Q)$ given by

$$
\begin{aligned}
F_{\lambda}\left([x, i]_{Q}\right):= & \begin{cases}{\left[\omega\left(x \cdot\left(\sigma_{Q}(x)+\frac{\sigma_{Q}(x)}{\varepsilon} \alpha(x)\right), 0\right]_{Q}\right.} & \text { if } i=1,0 \leq \sigma_{Q}(x) \leq \lambda \varepsilon, \\
{\left[x \cdot \frac{2 \varepsilon-\sigma_{Q}(x)}{\varepsilon}(\varepsilon+\alpha(x)) \frac{\lambda}{2-\infty}, 1\right]_{Q}} & \text { if } i=1, \lambda \varepsilon \leq \sigma_{Q}(x) \leq 2 \varepsilon, \\
{[x, 1]_{Q}} & \text { if } i=1, \sigma_{Q}(x) \geq 2 \varepsilon \\
{[x, 0]_{Q}} & \text { if } i=0 .\end{cases}
\end{aligned}
$$

Again, the map is well defined and continuous. Moreover, one easily verifies that $F_{0}=\operatorname{id}_{\mathbf{U}_{\omega}(Q)}$ and $F_{1}=\mathbf{U}_{\omega}\left(\iota_{P Q}\right) \circ f$, which means that $f$ is the right homotopy inverse of $\mathbf{U}_{\omega}\left(\iota_{P Q}\right)$. To show that it is also the left inverse first observe that the map $f$ also satisfies the formula

$$
\begin{aligned}
& f\left([x, i]_{Q}\right):= \\
& \qquad \begin{array}{ll}
{[\omega(x), 0]_{P}} & \text { if } i=1,0 \leq \sigma_{P}(x) \leq \alpha(x), \\
{\left[\omega\left(x \cdot\left(\sigma_{P}(x)+\frac{\sigma_{Q}(x)}{\varepsilon} \alpha(x)\right), 0\right]_{P}\right.} & \text { if } i=1, \alpha(x) \leq \sigma_{P}(x) \leq \varepsilon+\alpha(x), \\
{\left[x \cdot \frac{2 \varepsilon-\sigma_{Q}(x)}{\varepsilon}(\varepsilon+\alpha(x)), 1\right]_{P}} & \text { if } i=1, \varepsilon+\alpha(x) \leq \sigma_{P}(x) \leq 2 \varepsilon+\alpha(x) . \\
{[x, 1]_{P}} & \text { if } i=1, \sigma_{P}(x) \geq 2 \varepsilon+\alpha(x), \\
{[(x, 0)]_{P}} & \text { if } i=0,
\end{array}
\end{aligned}
$$

and consider the homotopy $G_{\lambda}: \mathbf{U}_{\omega}(P) \rightarrow \mathbf{U}_{\omega}(P)$ given by

$$
\begin{aligned}
& G_{\lambda}\left([x, i]_{Q}\right):= \\
& \begin{cases}{[\omega(x), 0]_{P}} & \text { if } i=1,0 \leq \sigma_{P}(x) \leq \lambda \alpha(x), \\
{\left[\omega\left(x \cdot \frac{\varepsilon+\alpha(x)}{\varepsilon}\left(\sigma_{P}(x)-\lambda \alpha(x)\right)\right), 0\right]_{P}} & \text { if } i=1, \lambda \alpha(x) \leq \sigma_{P}(x) \leq \lambda(\varepsilon+\alpha(x)), \\
{\left[x \cdot \frac{2 \varepsilon+\alpha(x)-\sigma_{P}(x)}{2 \varepsilon+\alpha(x)+\lambda(\varepsilon+\alpha(x))} \lambda(\varepsilon+\alpha(x)), 1\right]_{P}} & \text { if } i=1, \lambda(\varepsilon+\alpha(x)) \leq \sigma_{P}(x) \leq 2 \varepsilon+\alpha(x), \\
{[x, 1]_{P}} & \text { if } i=1, \sigma_{P}(x) \geq 2 \varepsilon+\alpha(x), \\
{[x, 0]_{P}} & \text { if } i=0 .\end{cases}
\end{aligned}
$$

One can verify that the map is well defined and continuous, $F_{0}=\operatorname{id}_{\mathbf{U}_{\omega}(P)}$ and $F_{1}=f \circ \mathbf{U}_{\omega}\left(\iota_{P Q}\right)$. This means that $f$ is a homotopy inverse of $\mathbf{U}_{\omega}\left(\iota_{P Q}\right)$. It follows from Proposition 3.3 that $f$ is a representative of the inverse of $\left[\mathbf{U}_{\omega\left(\iota_{P Q}\right)}\right]_{Z}$ in $\mathrm{Htp} \mathrm{Fib}_{Z}$, which proves the case when $P_{1}=Q_{1}$.

Assume in turn that $P_{2}=Q_{2}$. There exists a $T>0$ such that for every $x \in Q_{1}$ we have $x \cdot[0, T] \cap P_{2} \neq \varnothing$. Put $\beta(x):=\min \left(T, \sigma_{Q}(x)\right)$ and define the function $g: \mathbf{U}_{\omega}(Q) \rightarrow \mathbf{U}_{\omega}(P)$ by

$$
g\left([u, i]_{Q}\right):= \begin{cases}{[u \cdot \beta(u), 1]_{P}} & \text { if } i=1, \\ {[u, 0]_{P}} & \text { if } i=0 .\end{cases}
$$

One can easily verify that $g$ is a well defined continuous function. We will show that $g$ is a fiberwise deforming homotopy inverse of $\mathbf{U}_{\omega}\left(\iota_{P} Q\right)$. To this end consider the homotopy $F_{\lambda}: \mathbf{U}_{\omega}(Q) \rightarrow \mathbf{U}_{\omega}(Q)$ given by

$$
F_{\lambda}\left([u, i]_{Q}\right):= \begin{cases}{[u \cdot \lambda \beta(u), 1]_{Q}} & \text { if } i=1 \\ {[u, 0]_{Q}} & \text { if } i=0\end{cases}
$$


and the homotopy $G_{\lambda}: \mathbf{U}_{\omega}(P) \rightarrow \mathbf{U}_{\omega}(P)$ given by

$$
G_{\lambda}\left([u, i]_{P}\right):= \begin{cases}{[u \cdot \lambda \beta(u), 1]_{P}} & \text { if } i=1, \\ {[u, 0]_{P}} & \text { if } i=0 .\end{cases}
$$

One easily checks that both homotopies are well defined and join respectively $\operatorname{id}_{\mathbf{U}_{\omega}(Q)}$ with $\mathbf{U}_{\omega}\left(\iota_{P Q}\right) \circ g$ and $\operatorname{id}_{\mathbf{U}_{\omega}}\left(\iota_{P Q}\right)$ with $g \circ U_{\omega}\left(\iota_{P Q}\right)$. Thus Proposition 3.3 shows that $\mathbf{U}_{\omega(P)}$ and $\mathbf{U}_{\omega(Q)}$ have the same fiberwise deforming homotopy type. This completes the proof of Step 1.

Step 2. We lift the extra assumption. Put $R_{1}:=P_{1} \cup Q_{2}, R_{2}:=P_{1} \cap Q_{2}$. By Proposition 5.9 (ii), $\left(P_{1}, R_{2}\right)$ and $\left(R_{1}, Q_{2}\right)$ are index pairs. We have the commutative diagram of inclusions:

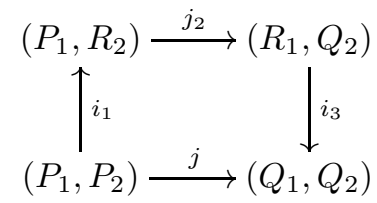

It is clear that the pair of index pairs $\left(P_{1}, P_{2}\right) \subset\left(P_{1}, R_{2}\right)$ satisfies the extra assumption of Step 1 and so does $\left(R_{1}, Q_{2}\right) \subset\left(Q_{1}, Q_{2}\right)$. Thus the inclusions $i_{1}$ and $i_{3}$ induce isomorphisms in Htp Fib $z$. Since $P_{1} \backslash R_{2}=P_{1} \backslash Q_{2}=R_{1} \backslash Q_{2}$, the inclusion $j_{2}$ induces an isomorphism by Proposition 4.3 .

Proof of Theorem 6.3. We need to show that if $M$ and $N$ are two isolating neighborhoods of $S, P$ a regular index pair for $N$ and $Q$ a regular index pair for $M$, then $\left[\mathbf{U}_{\omega}(P)\right]_{Z}$ and $\left[\mathbf{U}_{\omega}(Q)\right]_{Z}$ have the same fiberwise deforming homotopy type.

If $M=N$, then, by Proposition 5.9 (i), $P \cap Q$ is a regular index pair in $N$. Thus Theorem 6.2 may be applied to pairs $P \cap Q \subset P$ and $P \cap Q \subset Q$.

If $M \neq N$, one may always assume that $M \subset N$ since otherwise $M \cap N$ can be considered which is also an isolating neighborhood of $S$. Thus it is sufficient to show the existence of one regular index pair $P$ in $N$ and one regular index pair $Q$ in $M$ such that $\left[\mathbf{U}_{\omega}(P)\right]_{Z}$ and $\left[\mathbf{U}_{\omega}(Q)\right]_{Z}$ are equal.

By Theorem 5.7 there exists a regular index pair $P$ in $N$ such that $P_{1} \backslash P_{2} \subset$ int $M$. It is easily verified that $Q:=\left(M \cap P_{1}, M \cap P_{2}\right)$ is a regular index pair for $M$. Since $Q_{1} \backslash Q_{2}=M \cap\left(P_{1} \backslash P_{2}\right)=P_{1} \backslash P_{2}$, the inclusion $Q \subseteq P$ induces an isomorphism by Proposition 4.3 .

\section{The PRoperties of The Conley index Over A BASE}

We first prove the main properties of the Conley index over a base presented in Section 2

Proof of Theorem 2.2. Use the regular index pair $(\varnothing, \varnothing)$.

Proof of Theorem 2.3. One can choose disjoint isolating neighborhoods for $S$ and $S^{\prime}$, so the union is isolated. Now choose disjoint regular index pairs $P$ for $S$ and $P^{\prime}$ for $S^{\prime}$. The result follows from Proposition 4.12 .

Proof of Theorem 2.4. This is a consequence of Propositions 4.13 and 6.1.

Proof of Theorem 2.5. Obviously it is sufficient to show that for any $\lambda_{0} \in I$ there exists a neighborhood $\Delta_{0} \subset I$ of $\lambda_{0}$ such that $h_{\omega}\left(S^{\lambda}, \varphi^{\lambda}\right)$ does not depend on $\lambda \in \Delta_{0}$. Thus let us fix a $\lambda_{0} \in I$. We first introduce some notation. If $\Delta \subset I$ 
and $A \subset X \times I$, then we will write $A^{\Delta}$ for the set $A \cap X \times \Delta$ and we extend this notation in the obvious way to pairs, i.e. if $P=\left(P_{1}, P_{2}\right)$, then $P^{\Delta}:=\left(P_{1}^{\Delta}, P_{2}^{\Delta}\right)$.

For $\Delta \subset I$ there is a semiflow $\varphi^{\Delta}$ on $X \times \Delta$ given by

$$
\varphi^{\Delta}: X \times \Delta \times \mathrm{R}^{+} \ni((x, \lambda), t) \rightarrow\left(\varphi^{\lambda}(x, t), \lambda\right) \in X \times \Delta .
$$

Since

$$
\operatorname{Inv}\left(N \times \Delta, \varphi^{\Delta}\right)=\bigcup_{\lambda \in \Delta} S^{\lambda} \times\{\lambda\} \subset \bigcup_{\lambda \in \Delta} \operatorname{int} N \times\{\lambda\}=\operatorname{int}_{X \times \Delta} N \times \Delta,
$$

we see that $N \times \Delta$ is an isolating neighborhood with respect to $\varphi^{\Delta}$.

Let $M \subset N \times I$ be an isolating neighborhood of $\operatorname{Inv}\left(N \times I, \varphi^{I}\right)$ which admits a Lyapunov pair. Such an isolating neighborhood exists by Theorem 5.5. One easily verifies that if $\Delta \subset I$ and $P$ is a regular index pair in $M$ with respect to $\varphi^{I}$, then $P^{\Delta}$ is a regular index pair in $M^{\Delta}$ with respect to $\varphi^{\Delta}$. In particular, if $\lambda \in I$, then $P_{\lambda}$ is an index pair in $M^{\lambda}$ with respect to $\varphi^{\lambda}$.

Let $P, Q$ be regular index pairs in $M$ such that $P \subset \operatorname{int} Q$. We will show that there exists a neighborhood $\Delta$ of $\lambda_{0}$ such that if $\kappa, \lambda \in \Delta$, then $P^{\kappa} \subset Q^{\lambda}$. Indeed, if this is not true, then for some $i \in\{1,2\}$ there exist sequences $\lambda_{n}, \kappa_{n} \rightarrow \lambda_{0}$ and $x_{n} \rightarrow x \in N$ such that $x_{n} \in P_{i}^{\kappa_{n}} \backslash Q_{i}^{\lambda_{n}}$. It follows that $\left(x, \lambda_{0}\right) \in P_{i} \backslash \operatorname{int} Q_{i}$, a contradiction.

By Lemma 5.8 we can choose index pairs $P, Q, \bar{P}, \bar{Q}$ in $M$ such that $P \subset$ $\operatorname{int} Q, Q \subset \operatorname{int} \bar{P}, \bar{P} \subset \operatorname{int} \bar{Q}$. Choose $\Delta_{0}$, a neighborhood of $\lambda_{0}$ such that for $\lambda \in \Delta_{0}$

$$
P^{\lambda_{0}} \subset Q^{\lambda} \subset \bar{P}^{\lambda_{0}} \subset \bar{Q}^{\lambda}
$$

and let $\iota_{1}, \iota_{2}, \iota_{3}$ denote the corresponding inclusion maps. By Theorem 6.2 we have that $\mathbf{U}_{\omega}\left(\iota_{2} \iota_{1}\right)=\mathbf{U}_{\omega}\left(\iota_{2}\right) \mathbf{U}_{\omega}\left(\iota_{1}\right)$ and $\mathbf{U}_{\omega}\left(\iota_{3} \iota_{2}\right)=\mathbf{U}_{\omega}\left(\iota_{3}\right) \mathbf{U}_{\omega}\left(\iota_{2}\right)$ are isomorphisms. It follows that also $\mathbf{U}_{\omega}\left(\iota_{2}\right)$ is an isomorphism.

We conclude this section by presenting results on relations among the Conley indices over various base maps. In particular, by considering one-point space as a base, that results will also compare the classical index with the new one. We begin with the case of a constant base map $c_{z_{0}}$ for some $z_{0} \in Z$. The index $h_{c_{0}}(S, \phi)$, up to natural identifications, is equal to the (classical) wedge $h(S, \phi) \vee\left(Z, z_{0}\right)$, which we called in Section 4 the simple fiberwise deforming homotopy type. If $h_{\omega}(S, \varphi)$ is simple, then it does not contain any more information than the classical index provided that $Z$ is path connected.

Theorem 7.1. Suppose $N$ is a neighborhood for $S$ and $N$ is contractible in the phase space $X$. Then $h_{\omega}(S, \varphi)$ is simple.

Proof. This is a consequence of Corollary 4.7.

The following result states that $h_{\omega}(S, \varphi)$ depends on the homotopy class of $\omega$ only.

Theorem 7.2. If $\omega_{0}, \omega_{1}: X \rightarrow Z$ are continuous, and for some neighborhood $N$ of $S,\left.\left.\omega_{0}\right|_{N} \simeq \omega_{1}\right|_{N}: N \rightarrow Z$, then $h_{\omega_{0}}(S, \varphi)=h_{\omega_{1}}(S, \varphi)$.

Proof. This is a corollary from Proposition 4.6.

Let $S^{\prime}$ be another isolated invariant set for $\varphi$. 
Theorem 7.3. If $\zeta: Z \rightarrow Z^{\prime}$ is continuous, $Z^{\prime}$ is Hausdorff and $h_{\omega}(S, \varphi)=$ $h_{\omega}\left(S^{\prime}, \varphi\right)$, then $h_{\zeta \circ \omega}(S, \varphi)=h_{\zeta \circ \omega}\left(S^{\prime}, \varphi\right)$.

Proof. The result follows from Proposition 4.8

The following corollaries are easy consequences of Theorem 7.3 ;

Corollary 7.4. If $h_{\omega}(S, \varphi)=h_{\omega}\left(S^{\prime}, \varphi\right)$ for some $\omega$, then the classical Conley indices $h(S, \varphi)$ and $h\left(S^{\prime}, \varphi\right)$ are also equal each to the other.

Corollary 7.5. If $h_{\operatorname{id}_{X}}(S, \varphi)=h_{\operatorname{id}_{X}}\left(S^{\prime}, \varphi\right)$, then also $h_{\omega}(S, \varphi)=h_{\omega}\left(S^{\prime}, \varphi\right)$ for every map $\omega$.

As a consequence of Theorems 7.2 and 7.3 we obtain:

Corollary 7.6. If $\zeta: Z \rightarrow Z^{\prime}$ is a homotopy equivalence, $Z^{\prime}$ is a Hausdorff space, then $h_{\omega}(S, \varphi)=h_{\omega\left(S^{\prime}, \varphi\right)}$ if and only if $h_{\zeta \circ \omega}(S, \varphi)=h_{\zeta \circ \omega}\left(S^{\prime}, \varphi\right)$.

\section{EXAMPLES}

In the following examples the term "index" means $h_{\omega}(S)$ for a given isolated invariant set $S$. Here we use the simplest and most natural choices of $\omega$ and $Z$ to obtain information which is more delicate than that obtained by the classical index. We begin with our second example from the introduction.

Example 8.1. Suppose $X$ is the punctured plane, $\gamma_{1}$ is a homotopically nontrivial repelling periodic orbit, and $\gamma_{2}$ is a homotopically trivial repelling orbit. By Theorem 7.1 the index of $\gamma_{2}$ is simple, i.e. has the fiberwise deforming homotopy type of the one-point wedge of the classical Conley index of $\gamma_{1}$ and $Z$. In this case the classical index is the wedge of a 1 -sphere and a 2 -sphere. If we let $Z$ be the unit circle and $\omega$ be the map $z \mapsto z /|z|$, then the index of $\gamma_{1}$ over $Z$ has the homotopy type of the 2-torus. (This can be seen either by homotoping $Z$ to $\gamma_{1}$ and homotoping $\omega$ in a corresponding way, or by continuing $\gamma_{1}$ to a circle in the plane, and using an annulus as $P_{1}$.) Since $\gamma_{1}$ and $\gamma_{2}$ have different indices over $Z$, by Theorem [2.5, they are not related by continuation.

The next example shows that fiberwise deforming homotopy type is a finer classification than ordinary homotopy type.

Example 8.2. Again, suppose $X$ is the punctured plane, $Z$ is the unit circle and $\omega$ is the map $z \mapsto z /|z|$, but this time the periodic orbits are attracting, with $\gamma_{1}$ homotopically nontrivial and $\gamma_{2}$ homotopically trivial. We use annuli as the isolating neighborhoods and as $P_{1}$ in the index pair. In this example, since the exit set $P_{2}$ is empty, both indices over $Z$ have the same usual homotopy type, namely the disjoint union of two circles, one coming from $P_{1}$, the other from $Z$. However, the projections $r_{1}$ and $r_{2}$, for $\gamma_{1}$ and $\gamma_{2}$ respectively, map the annulus into different homotopy classes in $Z$. It follows from Proposition 3.5 that the resulting adjunction spaces are not fiberwise deforming homotopy equivalent, so again by Theorem 2.5. $\gamma_{1}$ and $\gamma_{2}$ are not related by continuation.

Example 8.3. If $x$ is $\mathrm{R}^{3}$ with the $z$-axis removed, $Z$ is the unit circle in the $x-y$ plane with the obvious map $\omega(x, y, z)=\left(x /\left(x^{2}+y^{2}\right), y /\left(x^{2}+y^{2}\right), 0\right)$, then the index over $Z$ will again distinguish periodic orbits in different homotopy classes. Let $\gamma$ be a hyperbolic periodic orbit winding $k$ times around the $z$-axis. Let $n=0,1,2$ be the dimension of the unstable manifold of the Poincaré map. If the Poincaré map 
preserves orientation on the unstable manifold, then the index is a locally trivial bundle over $S^{1}$ with the wedge of $k$ punctured spheres $S^{n}$ as the fiber. Moreover, the holonomy map transitively interchanges the spheres in the fiber and preserves their orientations. In particular, if $k=1$, then the index is $S^{n} \times S^{1}$; if $n=0$, then it is the disjoint union of two unit circles $S^{1}$ (one of them is treated as $Z$ ) and the projection $S^{1} \rightarrow Z$ (where $S^{1}$ denotes the other circle then $Z$ ) is given by $z \mapsto z^{k}$. If the Poincaré map reverses the orientation of the unstable manifold, then necessarily $n=1$. In the case $k=1$ the index is the Klein bottle; in general it is a locally trivial bundle over $S^{1}$ with the wedge of $k$ punctured circles $S^{1}$ as the

fiber and the holonomy map transitively interchanges the circles and reverses the orientation of exactly one circle.

\section{ACKNOWLEDGEMENT}

We thank Professor F. W. Lawvere for informing us about the literature on fiberwise pointed spaces.

\section{REFERENCES}

[Ba] T. Bartsch, The Conley index over a space, Math. Z. 209 (1992), 167-177. MR 92m:58019

[Bo] K. Borsuk, Theory of Retracts, PWN, Warsaw, 1967. MR 35:7306

[Ch] R. C. Churchill, Isolated invariant sets in compact metric spaces, J. Differential Equations 12 (1972), 330-352. MR 49:1536

[Co] C. Conley, Isolated Invariant Sets and the Morse Index, CBMS Lecture Notes, 38, Amer. Math. Soc., Providence, RI, 1978. MR 80c:58009]

[Coh] P. M. Cohn, The complement of a finitely generated direct summand of an abelian group, Proc. Amer. Math. Soc. 7 (1956), 520-521. MR 17:1182i

[E] R. Engelking, General Topology, Monografie Mathematyczne, Polish Scientific Publishers, Warsaw, 1977. MR 58:18316b; MR 84k:54035

[J1] I. M. James, General topology over a base, in: I. M. James, E. H. Kronheimer (editors), Aspects of Topology, Cambridge University Press, Cambridge, 1985. MR 86g:54031

[J2] I. M. James, General Topology and Homotopy Theory, Springer-Verlag, New York, Berlin, Heidelberg, Tokyo, 1984. MR 86d:55001

[J3] I. M. James, Fibrewise Topology, Cambridge Tracts in Math., 91, Cambridge University Press, Cambridge, 1989. MR 91a:55022

$[\mathrm{KM}]$ T. Kaczynski and M. Mrozek, Connected simple systems and the Conley functor, Top. Math. Nonlin. Anal. 10 (1997), 183-193. MR 99j:58134

[Ro] D. Rolfson, Knots and Links, Publish or Perish, Houston, 1990. MR 95c:57018

[Ry] K. P. Rybakowski, The Homotopy Index and Partial Differential Equations, SpringerVerlag, Berlin, Heidelberg, New York, 1987. MR 89d:58025

[Sm] J. Smoller, Shock Waves and Reaction-Diffusion Equations, Springer-Verlag, New York, Heidelberg, Berlin, 1983. MR 84d:35002 MR 91k:35130

[Sw] R. M. Switzer, Algebraic Topology - Homotopy and Homology, Springer-Verlag, New York, Heidelberg, Berlin, 1975. MR 52:6695

[W] E. A. Walker, Cancellation in direct sums of groups, Proc. Amer. Math. Soc. 7 (1956), 898-902. MR 18:403c

[Wh] G. W. Whitehead, Elements of Homotopy Theory, Graduate Texts in Math., 61, SpringerVerlag, New York, Heidelberg, Berlin, 1978. MR 80b:55001

Instytut Informatyki, Uniwersytet Jagielloński, 30-072 Kraków, Poland

E-mail address: mrozek@ii.uj.edu.pl

Department of Mathematics, SUNY at Buffalo, Buffalo, New York 14214-3093

E-mail address: reineck@newton.math.buffalo.edu

Instytut Matematyki, Uniwersytet Jagielloński, 30-059 Kraków, Poland

E-mail address: srzednic@im.uj.edu.pl 